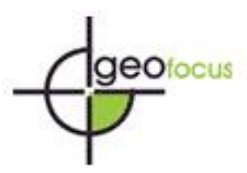

Olcina Cantos, J., Oliva Cañizares, A., Sánchez Almodóvar, E., Martí Talavera, J., Biener Camacho, S. (2021). Cartografías para la acreditación del riesgo de inundaciones: SNCZI y PATRICOVA en la Comunidad Valenciana (España). GeoFocus (Artículos), Revista Internacional de Ciencia y Tecnología de la Información Geográfica, 27, 19-53. http://dx.doi.org/10.21138/GF.691

\title{
CARTOGRAFÍAS PARA LA ACREDITACIÓN DEL RIESGO DE INUNDACIONES: SNCZI Y PATRICOVA EN LA COMUNIDAD VALENCIANA (ESPAÑA)
}

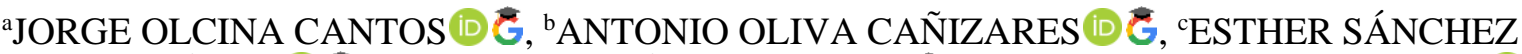

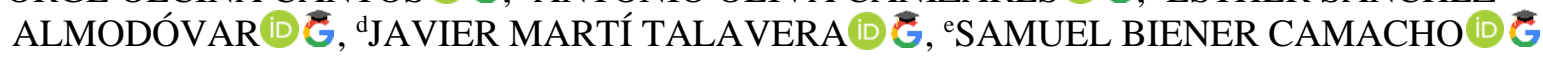 \\ Universidad de Alicante \\ Carretera Sant Vicent del Raspeig s/n 03690 Sant Vicent del Raspeig, España

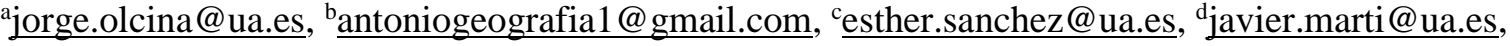 \\ samuel.biener@ua.es
}

\section{RESUMEN}

En España, las administraciones públicas con competencias en materia de ordenación del territorio y planificación urbanística no disponían hasta fechas recientes de una herramienta jurídica que acreditara el riesgo de inundación en un espacio geográfico. La Ley del Suelo 2/2008 (mod. 2015), resuelve este problema, puesto que obliga a realizar mapas de riesgos naturales como documento acreditativo de riesgo. En la actualidad, existen varias cartografías oficiales sobre inundaciones en el ámbito nacional y autonómico. En el caso que atañe al presente artículo, conviene destacar: el SNCZI (España) y el PATRICOVA (Comunidad Valenciana). Ambas cartografías son válidas a la hora de acreditar el riesgo de inundación; sin embargo, presentan algunas diferencias y similitudes que, en ocasiones, determinan la existencia de un riesgo en un territorio. Los resultados obtenidos muestran que el SNCZI prevalece por encima del PATRICOVA, empero, ambas cartografías son complementarias a la hora de determinar el riesgo de inundación. Por último, se propone una metodología novedosa para generar mapas de peligrosidad en función de la integridad física de una persona.

Palabras clave: Acreditación legal; cartografía oficial; riesgo de inundación; Vega Baja del Segura.

\section{CARTOGRAPHIES FOR THE ACCREDITATION OF FLOOD RISK: SNCZI AND PATRICOVA IN COMUNIDAD VALENCIANA (SPAIN).}

\begin{abstract}
In Spain, the public administrations with powers in the land use and urban planning lacked a legal tool to validate the risk of flooding in a geographical zone. The Land Law 2/2008 (mod. 2015), solves this problem since it forces to develop natural risk maps as a document accrediting risk. Currently, there are Recepción: 22/02/2021

Aceptación definitiva: 23/04/2021

Editor al cargo: Dr. Pere Serra

Attribution-NonCommercial-NoDerivatives 4.0 International (CC BY-NC-ND 4.0) 
Olcina Cantos, J., Oliva Cañizares, A., Sánchez Almodóvar, E., Martí Talavera, J., Biener Camacho, S. (2021). Cartografías para la acreditación del riesgo de inundaciones: SNCZI y PATRICOVA en la Comunidad Valenciana (España). GeoFocus (Artículos), Revista Internacional de Ciencia y Tecnología de la Información Geográfica, 27, 19-53. http://dx.doi.org/10.21138/GF.691

several official cartographies on floods at a national and regional level. Nowadays, there are different official cartographies in which both national and autonomous floods appear. In this article, there are two outstanding: SNCZI (Spain) and PATRICOVA (Comunidad Valenciana). Both maps are valid when it comes to proving flood risk. However, they present some differences and similarities that determine the existence of a risk in a territory. The results showed that the SNCZI prevails over the PATRICOVA, however both maps are complementary when it comes to determining flood risk. Finally, a innovative methodology is proposed to generate hazard maps based on the physical integrity of a person.

Keywords: Legal validation; official cartography; flood risk; Vega Baja del Segura.

\section{Introducción}

La cartografía es una de las medidas más eficaces para la reducción del riesgo natural. Su utilización en los procesos de ordenación del territorio permite delimitar áreas de grado diverso de riesgo y evitar la ocupación con nuevos usos de aquellas zonas con elevado riesgo. La elaboración de cartografías de riesgos naturales ha experimentado un avance muy notable en los últimos años en relación con la aplicación de modernas tecnologías cartográficas (Olcina \& Oliva, 2020). Los mapas ayudan a la comprensión, difusión y comunicación de información geográfica, dirigida tanto a la población como a los técnicos de las administraciones públicas. Se trata de una herramienta que permite, con un solo golpe de vista, identificar la información que se está representando, a la vez que de la temática que se trata.

Davoine et al. (2020) señalan que el mapa es un instrumento esencial para difundir y comunicar información geográfica que, al incorporar contenido multimedia, se convierte en un nuevo medio para comunicar, comprender y reflexionar sobre datos espaciales, temporales e históricos. Asimismo, indican que existen estudios que muestran la importancia del formato cartográfico como vector de difusión de información.

La comunicación del riesgo de inundación desempeña un papel importante en la gestión de riesgos, porque puede fortalecer la conciencia de las personas ante el riesgo y puede motivarlas a tomar medidas de precaución y, por ello, los mapas se convierten en una herramienta básica de representación e información geográfica (Kellens et al., 2009).

Kellens et al. (2009) señalan que los mapas de peligro o riesgo de inundación pueden servir de base para la planificación espacial, el peligro local, evaluación, planificación de emergencias, medidas técnicas de protección y aumento del riesgo en la conciencia pública, etc. (EXCIMAP, 2007). Además, los propios mapas pueden contener información de vital importancia para los habitantes, que residen en las zonas propensas a las inundaciones (Kellens et al., 2009), que desconocen dónde pueden consultar los mapas y conocer si sus viviendas se hallan en espacios inundables o no.

Mientras que los mapas de peligrosidad de inundación contienen información sobre la probabilidad y/o magnitud de una inundación, los mapas de riesgo de inundación muestran información adicional sobre sus consecuencias, por ejemplo, daños económicos y víctimas (Kellens et al., 2009), a los que hay que añadir, en la actualidad, los impactos ambientales y patrimoniales. Sin olvidar que estos mapas son dinámicos, especialmente en el actual contexto de cambio climático. 
Olcina Cantos, J., Oliva Cañizares, A., Sánchez Almodóvar, E., Martí Talavera, J., Biener Camacho, S. (2021). Cartografías para la acreditación del riesgo de inundaciones: SNCZI y PATRICOVA en la Comunidad Valenciana (España). GeoFocus (Artículos), Revista Internacional de Ciencia y Tecnología de la Información Geográfica, 27, 19-53. http://dx.doi.org/10.21138/GF.691

En regiones con elevado riesgo natural, como el litoral mediterráneo español, el uso de mapas de riesgo en la planificación territorial se convierte en un instrumento necesario para la minimización del riesgo. Sin olvidar que, desde 2008, la legislación del suelo obliga a utilizar los mapas como documento jurídico de acreditación del riesgo en los procesos de asignación de nuevos usos en el territorio. Algunas Comunidades Autónomas en nuestro país (País Vasco, Baleares, C. Valenciana, Cataluña o Andalucía), obligaban con anterioridad a dicha fecha, a la consulta de cartografías de inundación a la hora de redactar planes urbanísticos, debido a la existencia de planes regionales -sectoriales- de reducción del riesgo de dicho peligro natural mediante la ordenación territorial.

El riesgo de inundación en el litoral mediterráneo, aspecto consustancial a esta región geográfica, se ha incrementado en las últimas décadas en relación con el aumento de la vulnerabilidad y exposición al peligro de lluvias intensas. Ello en relación con la intensa transformación del territorio por construcciones antrópicas, han terminado ocupando espacios inundables, incrementando de manera exponencial los daños económicos y generando, asimismo, víctimas humanas. Frente a las soluciones habituales llevadas a cabo en nuestro país que han tenido en las costosas -y muchas veces ineficaces-, obras hidráulicas la piedra angular de las políticas de reducción del riesgo. Por este motivo, la elaboración de mapas de riesgo se convierte en una herramienta fundamental para la ordenación del territorio, regulación de usos y la salvaguarda de las vidas humanas (Olcina \& Oliva, 2020), a la vez que se plantea como base para la redacción de Planes de Emergencia a escala municipal y regional (Oliva et al. 2020), así como para la gestión de la propia emergencia (Camarasa \& Caballero, 2018).

\subsection{Evolución del marco jurídico}

El mapa se ha convertido en el documento jurídico de acreditación del riesgo en España, dentro del contexto de planificación territorial. En ello se ha seguido el ejemplo de algunos países europeos que, en las últimas décadas, han desarrollado normativa y planes para la reducción del riesgo a partir de medidas "blandas" basadas en la ordenación del territorio y la confección de cartografía de riesgo (Francia, Alemania, Reino Unido, Italia). El impulso decisivo en esta línea ha venido tras la promulgación de la Directiva 60/2007 de gestión del riesgo de inundación, que recogió el principio de precaución del Tratado de Funcionamiento de la UE (art. 191), como fundamento jurídico para la reducción de riesgo y la protección sostenible del territorio.

Debe recordarse que en España el año 1996, tras el desastre de Biescas, marca un cambio de rumbo en las políticas de reducción del riesgo de inundaciones en España y su plasmación normativa. La modificación de la Ley del Suelo de 1992, con la aprobación de la nueva ley de 1998, que incluía un artículo orientado a impedir la ocupación con uso urbano de espacios con riesgo natural suponía una modificación en las propuestas en materia hidráulica, basadas básicamente en la obra de ingeniería. No obstante, esta ley del suelo de 1998, nunca se cumplió en su artículo 13, porque los municipios aducían falta de instrumentos jurídicos para acreditar el riesgo natural y, por tanto, clasificar como no urbanizable los territorios con riesgo (Olcina, 2018). Solo a partir de la ley del suelo de 2008, revisada en 2015, ha sido posible determinar que el documento de acreditación del riesgo natural es el mapa. El artículo 23 ha fijado esta cuestión de manera manifiesta. En la actualidad cualquier proceso de transformación urbana del suelo debe ir acompañado de un mapa de riesgos naturales, de todos los existentes en la zona objeto de transformación. Esta acción jurídica ha tenido tres procesos paralelos, asimismo destacados y necesarios 


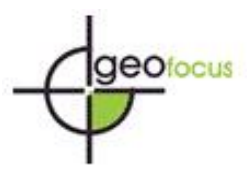

Olcina Cantos, J., Oliva Cañizares, A., Sánchez Almodóvar, E., Martí Talavera, J., Biener Camacho, S. (2021). Cartografías para la acreditación del riesgo de inundaciones: SNCZI y PATRICOVA en la Comunidad Valenciana (España). GeoFocus (Artículos), Revista Internacional de Ciencia y Tecnología de la Información Geográfica, 27, 19-53. http://dx.doi.org/10.21138/GF.691

para el cambio de procedimientos de reducción del riesgo de inundación mediante la ordenación del territorio.

En este contexto, resultó muy importante la promulgación del RD 638/2016, por el que se modifica el Reglamento de Dominio Público Hidráulico (DPH), y donde se introducen novedades importantes para la consideración y tratamiento del riesgo de inundaciones en la planificación territorial (Perles et al., 2018). Destaca en esta norma la ampliación de criterios para la delimitación del DPH, sus áreas de influencia y las zonas inundables que a partir de ahora permite el uso de fuentes y métodos hidrológicos, hidráulicos, geomorfológicos, fotográficos, cartográficos e históricos.

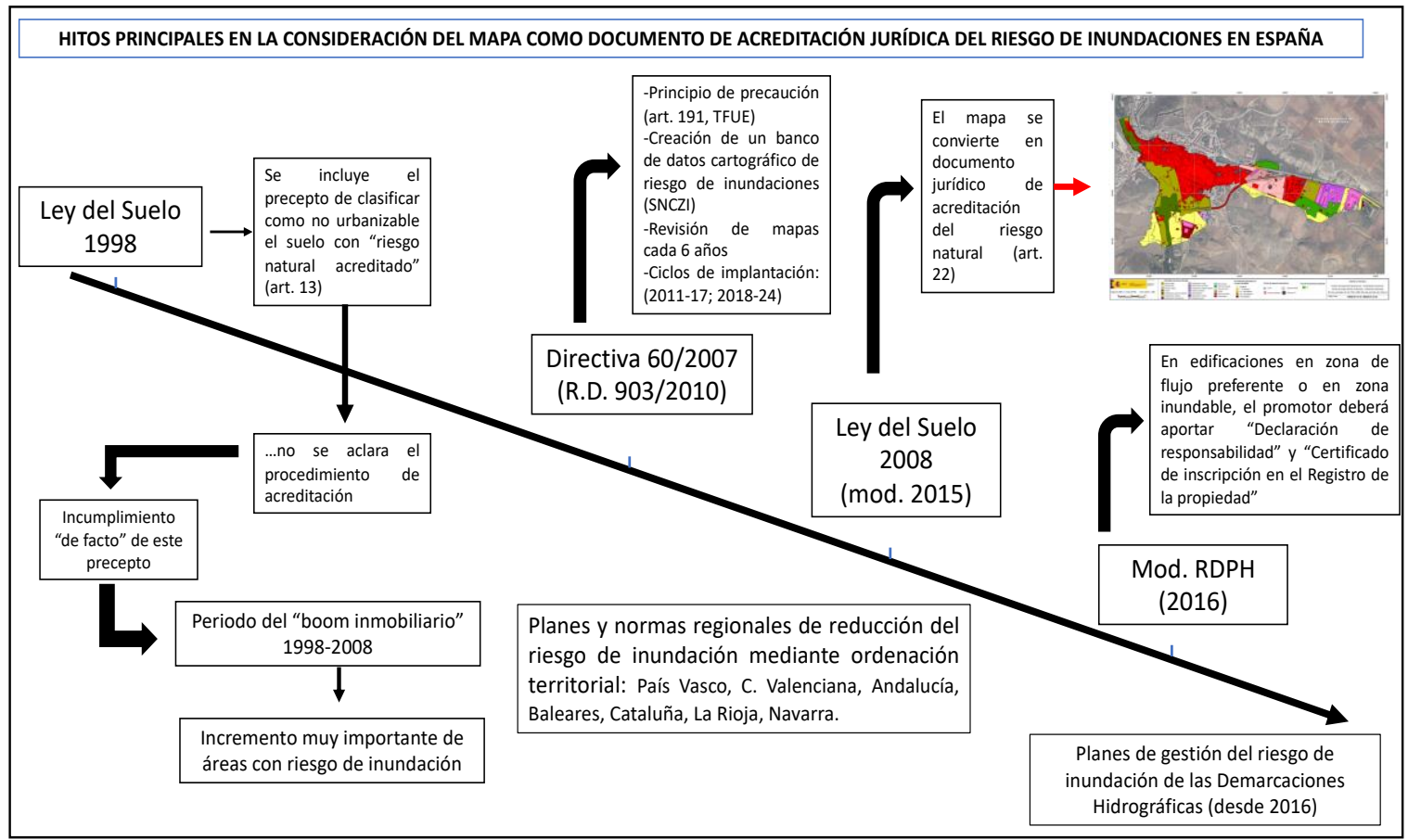

Figura 1. Proceso de incorporación del mapa como documento de acreditación jurídica del riesgo de inundación en España.

Fte.: Elaboración propia.

Al amparo de la Directiva 60/2007 se ha producido un tránsito, importante y necesario, en la confección y utilización de los mapas de inundaciones en los procesos territoriales: se ha pasado de los mapas de peligrosidad, elemento primero pero no único para la determinación del grado de importancia del fenómeno en un territorio, a los mapas de riesgo, que combinan peligrosidad y vulnerabilidad, esto es, presencia y usos del ser humano en el territorio incluyendo, asimismo, el impacto de las inundaciones en el medio natural. Debe quedar claro que un mapa de riesgo de inundación es un documento clave para la clasificación urbanística, de ahí que su elaboración requiera precisión y detalle. No es un mapa de peligrosidad, como ha sido habitual elaborar en los análisis del riesgo con finalidad urbanística; el mapa de riesgo incluye peligrosidad y agrega la vulnerabilidad social, económica y patrimonial. La escala de 


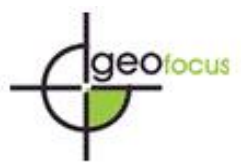

Olcina Cantos, J., Oliva Cañizares, A., Sánchez Almodóvar, E., Martí Talavera, J., Biener Camacho, S. (2021). Cartografías para la acreditación del riesgo de inundaciones: SNCZI y PATRICOVA en la Comunidad Valenciana (España). GeoFocus (Artículos), Revista Internacional de Ciencia y Tecnología de la Información Geográfica, 27, 19-53. http://dx.doi.org/10.21138/GF.691

elaboración debe adaptarse a las tramas urbanas y para ello es necesario, además de ajustes cartográficos, la realización de trabajo de campo para confirmar los resultados que ofrecen los portales cartográficos de las administraciones (Olcina \& Díez, 2017).

Los antecedentes normativos que justifican la necesidad de elaboración de cartografías de riesgo de inundación surgen con la aprobación de la Ley 2/1985, de 21 de enero, sobre Protección Civil, en la cual se establece la necesidad de proteger a las personas y los bienes a través de una adecuada y previa planificación ante situaciones de grave riesgo, catástrofe o calamidad; en la cual las inundaciones pueden dar lugar a este tipo de situaciones. Asimismo, el Real Decreto 407/1992, de 24 de abril, se aprueba la Norma Básica de Protección Civil, prevista en el artículo 8 de la anterior Ley citada. Así pues, en dicho Real Decreto, se dispone que serán objeto de planes especiales, entre otros, las emergencias que pueden derivarse de inundaciones y que estos planes serán elaborados de acuerdo con la correspondiente Directriz Básica que aprobase el Gobierno y que, debería establecer los fundamentos comunes y los requisitos mínimos sobre organización, criterio operativo, medidas de intervención e instrumentos de coordinación que deben reunir dichos planes.

Por otra parte, tras las inundaciones acontecidas en el año 2004 en territorio europeo, se aprueba, por el Parlamento Europeo y el Consejo de la Unión Europea, la Directiva 60/2007/CE sobre la evaluación y gestión de los riesgos de inundación, la cual señala que las inundaciones no pueden evitarse y causan daños sociales, económicos y ambientales; y que bajo el contexto del cambio climático en la cual serán más recurrentes, conviene tomar medidas paliativas de las mismas. Para ello, la Directiva señala que <<es necesario elaborar mapas de peligrosidad y riesgo de inundación, en la cual se desarrollarán los planes de gestión del riesgo de inundación [...] >>. Los mapas de peligrosidad y riesgo <<deben realizarse a la escala que resulte más apropiada >. La trasposición de la Directiva en el marco jurídico español por medio del Real Decreto 903/2010, de 9 de julio, del mismo nombre, contempla las mismas cuestiones y señala la importancia de la elaboración de mapas de peligrosidad y de riesgo como medida de reducción de los efectos negativos de las inundaciones, regulando los usos y previniendo la ocupación en zonas inundables.

Posteriormente a la aprobación de la Directiva, y antes de su transposición al marco jurídico español, se aprueba en España el Real Decreto 9/2008, de 11 de marzo, por el que se modifica el Reglamento del Dominio Público Hidráulico (RDPH), indicando que no es el documento de trasposición pero que recoge los ideales establecidos en la Directiva, donde se indica que <<existe una necesidad de incorporar una herramienta que permita actuar eficazmente y que se encuentre a disposición de los ciudadanos la delimitación cartográfica del Dominio Público Hidráulico, Zona de Flujo Preferente y Zonas Inundables, con un efecto preventivo >>. Para ello, se plantea la elaboración de un Sistema Nacional de Cartografía de Zona Inundables (SNCZI), a desarrollar <<en colaboración con las Comunidades Autónomas, que aportarán una información muy valiosa, para que se tenga en cuenta por las restantes administraciones en el ejercicio de sus competencias en ordenación del territorio y planificación urbanística, y que será imprescindible para incrementar la seguridad de los ciudadanos >>. Dicha cartografía, se basa en <<la máxima crecida ordinaria que se ha mostrado claramente insuficiente en numerosas situaciones, por lo que resulta imprescindible que los cauces naturales se definen no sólo por criterios hidrológicos, sino atendiendo también a características, como las geomorfológicas, ecológicas y las referencias históricas disponibles $>>$, tales como las analizadas en diversos estudios referentes a esta área (Conesa, 1995; CHS, 2020; Romero \& Maurandi, 2000; Sanchez et al., 1989). No obstante, algunos autores señalan que la mayor parte de las cartografías de inundabilidad recogidas en el SNCZI no se han 
Olcina Cantos, J., Oliva Cañizares, A., Sánchez Almodóvar, E., Martí Talavera, J., Biener Camacho, S. (2021). Cartografías para la acreditación del riesgo de inundaciones: SNCZI y PATRICOVA en la Comunidad Valenciana (España). GeoFocus (Artículos), Revista Internacional de Ciencia y Tecnología de la Información Geográfica, 27, 19-53. http://dx.doi.org/10.21138/GF.691

considerado los criterios geomorfológicos recomendados en la delimitación de las áreas inundables, puesto que utilizan o se rigen por criterios hidrológicos-hidráulicos (Soriano, Sanchis \& Camarasa, 2019; Gallegos \& Perles, 2020), mientras que el PATRICOVA, tras su revisión, sí que incluye elementos geomorfológicos. En Reino Unido, dicha cuestión ha sido objeto de diversas investigaciones en las que se ha argumentado la importancia que tiene la participación de la ciudadanía local, cuestionando la ejecución de modelos hidrológicos-hidráulicos, para la confección de la cartografía de inundación (Whatmore \& Landström, 2011) y poniendo de relieve la necesidad de conocer el territorio sobre el que se ha asentado la población, que ha dado lugar a paisajes de riesgo de complicada gestión (Lane et al., 2011; Whatmore, 2009).

En lo que respecta al ámbito nacional español, se han ido aprobando tanto Planes de Ordenación del Territorio para la reducción del Riesgo de Inundación, como Planes de Protección Civil que incluyen cartografía de riesgo. En el caso de los primeros, el País Vasco, Baleares y Canarias, Andalucía y Cataluña, poseen sus propios planes de ordenación con cartografía de riesgo: PTS de Ordenación de los ríos y arroyos, Directrices y Planes Insulares de Ordenación del Territorio que incluyen mapas adaptados a su ley del suelo no urbanizable, Plan de prevención de avenidas e inundaciones en Cauces Urbanos Andaluces, Planificación de los espacios fluviales y prevención de las inundaciones, respectivamente.

Por su parte, en el ámbito regional, existen planes de protección civil que incluyen cartografía de riesgo -básicamente de peligrosidad- como Plan Especial de Emergencias ante el Riesgo de Inundaciones de la Comunidad Autónoma del País Vasco, INUNBAL (Baleares), Plan de Emergencia de Fenómenos Meteorológicos Adversos (Baleares), Plan de Emergencia ante el Riesgo de Inundaciones en Andalucía, INUNCAT (Cataluña).

Como se ha señalado, es a partir del RD 2/2008 cuando realmente las administraciones (local y regional) disponen de una herramienta legal para exigir el cumplimiento de la elaboración de un mapa de riesgo natural (inundación) para cualquier actuación urbanística. No obstante, la realización del SNCZI, por imperativo de la Directiva 60/2007, junto a la existencia señalada de cartografía oficial de riesgo de inundación en algunas Comunidades Autónomas, ha favorecido interpretaciones intencionadas en la escala local sobre la prioridad de uso de unos mapas oficiales u otros a la hora de su manejo en los procesos de planificación territorial. Este hecho se ha puesto de manifiesto en la C. Valenciana, a raíz de recientes eventos de inundación ocurridos, cuyos efectos han evidenciado la realidad de una interpretación muy permisiva de las normativas del suelo y del territorio a la hora de limitar o adecuar actuaciones urbanísticas a la realidad del riesgo existente.

De ahí que sea necesario aclarar esta cuestión a partir del análisis de los criterios de elaboración de las cartografías oficiales de riesgo de inundación y de la aclaración de la jerarquía legal de aplicación de ellas en la ordenación territorial.

\subsection{Hipótesis y objetivos}

Es necesario realizar un análisis comparativo de las cartografías oficiales (SNCZI y PATRICOVA) y aclarar la prioridad, con base jurídica, de uso de una y otra, puesto que el mapa de inundaciones se presenta como la principal herramienta de medidas de prevención basadas en la ordenación del territorio y como alternativa a las soluciones tradicionales -obras duras- en el ámbito nacional. Además, se plantea la 
Olcina Cantos, J., Oliva Cañizares, A., Sánchez Almodóvar, E., Martí Talavera, J., Biener Camacho, S. (2021). Cartografías para la acreditación del riesgo de inundaciones: SNCZI y PATRICOVA en la Comunidad Valenciana (España). GeoFocus (Artículos), Revista Internacional de Ciencia y Tecnología de la Información Geográfica, 27, 19-53. http://dx.doi.org/10.21138/GF.691

necesidad de realizar estudios de inundación de mayor detalle, a escala municipal o inferior, para determinar el nivel de riesgo de inundación real para cada municipio.

Las hipótesis de investigación pretenden responder dos cuestiones principales:

- ¿Por qué existen diferencias entre la cartografía de riesgo de inundación entre el PATRICOVA y el SNCZI?

-A efectos urbanísticos, ¿Cuál de estas dos cartografías debe contemplarse como documento de acreditación jurídica del riesgo de inundación para garantizar la vida de las personas? Objetivo principal de los análisis de riesgo.

Por consiguiente, los objetivos a alcanzar en la presente investigación son:

a) Realizar una comparativa entre el PATRICOVA y el SNCZI en un espacio geográfico de elevado riesgo: la comarca de la Vega Baja del Segura.

b) Delimitar áreas de conflicto en relación con los usos del suelo existentes

c) Realizar propuestas para que se tengan en cuenta en las próximas revisiones de ambas cartografías oficiales.

Para ello, es necesario realizar un análisis comparativo entre el SNCZI y el PATRICOVA, en lo que se refiere a la metodología empleada y a los resultados cartográficos. La zona de estudio seleccionada es la Vega Baja del Segura sensu stricto, en la provincia de Alicante, hecho que justifica que los ejemplos anteriormente señalados en este apartado sean de esta región. Este espacio se ha escogido por varios motivos: en primer lugar, la inundación de septiembre de 2019 plantea realizar un estudio de inundación más detallado en la Vega Baja, a escala municipal; en segundo lugar, como se ha señalado anteriormente, los mapas regionales suelen dar ciertos errores y problemas en función de la escala empleada en los sectores del río Segura y Tajo; y en tercer lugar, actualmente se ha redactado el informe de riesgos ambientales del Plan de Actuación Territorial (PAT) de la Vega Baja, a la vez que se está realizando un estudio de inundabilidad en la Vega Baja por la Universidad Politécnica de Valencia para dicho PAT. Asimismo, la redacción de este artículo se realiza en el año 2021, a dos años para la nueva revisión del PATRICOVA, por lo que este artículo pretende realizar determinadas observaciones, valoraciones y propuestas que pueda tenerse en cuenta en la nueva revisión de este plan normativo sectorial.

\section{Materiales, datos y métodos}

\section{1 Ámbito de estudio.}

El ámbito de estudio abarca la denominada Vega Baja del Segura, ubicada en la comarca más meridional de la provincia de Alicante y de la Comunidad Valenciana: el Bajo Segura (véase Figura 2). Se trata de la llanura de inundación del río Segura que, en septiembre de 2019, el río se desbordó tras un episodio de lluvias torrenciales que superaron $\operatorname{los} 500 \mathrm{l} / \mathrm{m}^{2}$ en algunos puntos de la comarca, lo que provocó una crecida de las aguas del río que terminaron por producir varias roturas de las motos del muro de encauzamiento, cuya consecuencia fue la anegación de gran parte de la Vega Baja. Los daños económicos fueron elevados y, asimismo, se produjeron la pérdida de 3 vidas humanas, al igual que en 1987, lo que significa que a pesar del Plan de Defensa contra Avenidas de la Cuenca del Segura no se ha conseguido reducir el riesgo de inundación, sino que se ha incrementado a causa de la mayor exposición 
Olcina Cantos, J., Oliva Cañizares, A., Sánchez Almodóvar, E., Martí Talavera, J., Biener Camacho, S. (2021). Cartografías para la acreditación del riesgo de inundaciones: SNCZI y PATRICOVA en la Comunidad Valenciana (España). GeoFocus (Artículos), Revista Internacional de Ciencia y Tecnología de la Información Geográfica, 27, 19-53. http://dx.doi.org/10.21138/GF.691

por parte del hombre. Bien sea por cuestiones económicas o por la falsa seguridad que ha generado dichas obras que han resultado ser ineficaces.

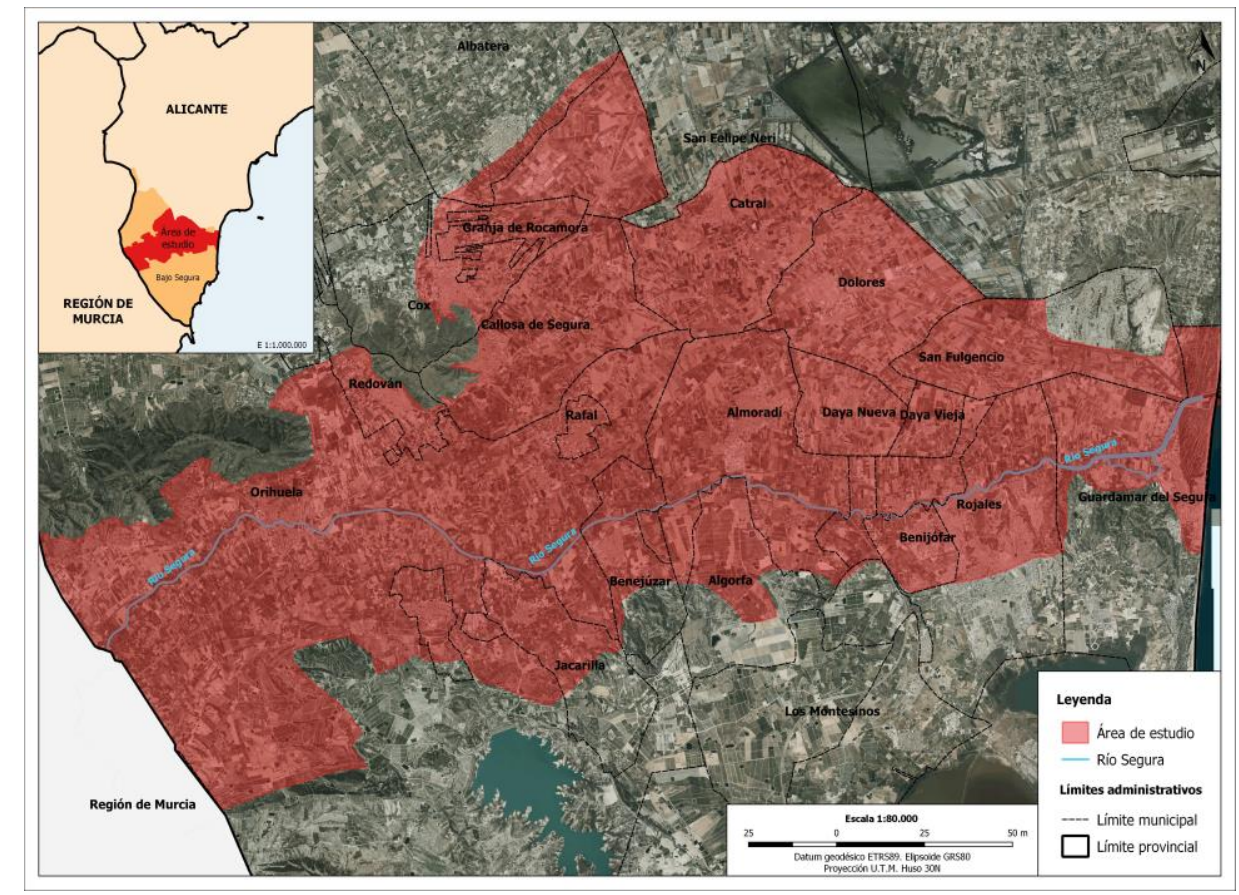

Figura 2. Zona de estudio: la Vega Baja del Segura.

Fte.: Elaboración propia.

El significado de Vega está estrechamente relacionado con la huerta que ocupa el espacio geográfico de la llanura de inundación del río Segura, en su tramo más bajo. Bajo este criterio -la llanura de inundación del río Segura- se ha seleccionado como zona de estudio la Vega Baja del Segura, sensu stricto, a consecuencia del episodio de inundación acaecido en septiembre de 2019, en tierras alicantinas. Esto supone que la zona de estudio ocupa la huerta desde la entrada del río Segura en el término municipal de Orihuela hasta su desembocadura en Guardamar del Segura, ajustándose al espacio mencionado. La zona de estudio seleccionada presenta una superficie total de $302,22 \mathrm{~km}^{2}$ que abarca, en gran medida, la llanura de inundación del río Segura. Este hecho supone un problema en la representación cartográfica a nivel escalar, lo que implica que para tener una visión global en conjunto (1:80 000), se pierde información geográfica por la generalización. Para solventar este problema, en relación con la finalidad de la investigación, en el apartado de Descripción y análisis de los resultados, se modifica la escala de trabajo al ámbito local para poder apreciar más detalladamente y de manera precisa, las diferencias existentes entre el SNCZI y el PATRICOVA. 
Olcina Cantos, J., Oliva Cañizares, A., Sánchez Almodóvar, E., Martí Talavera, J., Biener Camacho, S. (2021). Cartografías para la acreditación del riesgo de inundaciones: SNCZI y PATRICOVA en la Comunidad Valenciana (España). GeoFocus (Artículos), Revista Internacional de Ciencia y Tecnología de la Información Geográfica, 27, 19-53. http://dx.doi.org/10.21138/GF.691

\subsection{Datos}

Para llevar a cabo este trabajo se han tenido en cuenta investigaciones que presentan objetivos similares al que muestra el presente estudio, como es, por ejemplo, el desarrollado por Soriano, Sanchis \& Camarasa (2020), denominado Integración de cartografías de inundabilidad en la Comunitat Valenciana (PATRICOVA y SNCZI), en el cual el objetivo principal es crear un mapa que represente la peligrosidad y riesgo de inundación fusionando ambas cartografías, incluyendo el análisis de las edificaciones ubicadas dentro o fuera de dichas zonas.

Los geodatos empleados para la realización del presente análisis se corresponden con la cartografía en formato vectorial (shapefile) a escala 1:25000 relativa al Sistema Nacional de Cartografía de Zonas Inundables (SNCZI) y al Plan de Acción Territorial de Carácter Sectorial sobre Prevención del Riesgo de Inundación en la Comunidad Valenciana (PATRICOVA). En lo que respecta al SNCZI, se han obtenido, a través de la IDE del Ministerio para la Transición Ecológica y el Reto Demográfico, cuatro capas de información geográfica en formato vectorial que representan las Zonas Inundables según los periodos de retorno (T) establecidos en 10, 50, 100 y 500 años. Esta cartografía determina, según el periodo de retorno, el nivel alcanzado por la lámina de agua y la extensión de área inundada, y la cartografía de riesgo para los T10, T100 y T500. En cuanto al PATRICOVA, se adquiere, mediante el Instituto Cartográfico Valenciano (ICV) de la Conselleria de Urbanismo, Obras Públicas y Vertebración del Territorio, la capa en formato vectorial correspondiente a la peligrosidad por inundación que representan los seis niveles de peligrosidad según frecuencia de inundación (baja, media y alta) y los calados (inferior o superior a 0.80 $\mathrm{m}$ ), e incorpora el nivel de peligrosidad geomorfológica, compuesto por la red de cauces de la cartografía oficial del Instituto Cartográfico Valenciano (1:10 000) y/o aquellos que tienen una cuenca vertiente superior a $0,5 \mathrm{~km}^{2}$, y la capa de riesgo de inundación que representa los cinco niveles de riesgo (Muy Bajo, Bajo, Medio, Alto y Muy Alto).

Para el análisis cartográfico se ha empleado la cartografía base (CV05) a escala 1:5000 proporcionada por el ICV, de la cual se han extraído las edificaciones correspondientes a los municipios que se encuentran en la zona de estudio delimitada. De los geoprocesos realizados con las capas de información antes mencionadas, se han obtenido varios resultados que muestran, por un lado, las diferencias existentes entre ambos sistemas de cartografía en cuanto al análisis de la peligrosidad, y de otro lado, se pone de manifiesto las construcciones existentes afectadas por peligrosidad y riesgo de inundación.

\subsection{Materiales y Técnicas de análisis.}

La metodología empleada para la presente investigación es bastante extensa por lo que se exponen los aspectos más generales para poder matizar, en mayor medida, aquellos aspectos más relevantes que han permitido desarrollar el presente trabajo.

En primer lugar, se ha procedido a realizar la consulta de bibliografía internacional y nacional con la finalidad de conocer aquellos estudios que ponen de manifiesto la importancia de la cartografía o de la cartografía de riesgo para la sociedad y, sobre todo, como documentos de acreditación en el territorio para aspectos relacionados en materia de ordenación y planificación urbanística. Sin olvidar, la importancia que presentan estos mapas en la normativa de Protección Civil. Para apoyar a las aportaciones científicas, se 


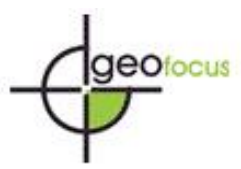

Olcina Cantos, J., Oliva Cañizares, A., Sánchez Almodóvar, E., Martí Talavera, J., Biener Camacho, S. (2021). Cartografías para la acreditación del riesgo de inundaciones: SNCZI y PATRICOVA en la Comunidad Valenciana (España). GeoFocus (Artículos), Revista Internacional de Ciencia y Tecnología de la Información Geográfica, 27, 19-53. http://dx.doi.org/10.21138/GF.691

realiza un breve análisis evolutivo sobre las normativas, desde la escala europea hasta la local, sobre la importancia de la cartografía de riesgo para las normativas concretas sobre inundación, así como las normativas del suelo. Para alcanzar los objetivos planteados en el anterior apartado y dar respuesta a la primera pregunta planteada en la hipótesis de trabajo, se hace necesario realizar un análisis metodológico del SNCZI y el PATRICOVA que, en primera instancia, permite conocer por qué ambas cartografías presentan semejanzas y diferencias, y a qué se deben estas últimas.

Antes de comentar la base metodológica sobre la que se basa el Sistema Nacional de Cartografía de Zonas Inundables (SNCZI), es necesario establecer unos antecedentes. Como ya se ha comentado, la realización de mapas de peligrosidad y riesgo de inundación en España, tienen su origen en el cumplimiento de la Directiva 2007/60/CE. Dicha norma establece tres fases para su implantación: Evaluación Preliminar del Riesgo de Inundación (EPRI), elaboración de Mapas de Peligrosidad y Riesgo de Inundación (MPRI) y la redacción de los Planes de Gestión de Riesgo de Inundación (PGRI) (CHS, 2019). Para la realización de los mapas de peligrosidad se toma como base la información aportada por el Modelo Digital de Elevaciones (MDE) que, tras los tratamientos necesarios, se utiliza para realizar los estudios hidrológicos e hidráulicos pertinentes. En el primero de los casos, es necesario comentar que el modelo hidrológico se ejecuta tanto en régimen natural como en régimen alterado (CHS, 2019). De tal manera que se obtienen los caudales de la máxima crecida ordinaria, así como las asociadas a los periodos de retorno de 10, 25, 50, 100 y 500 años. Para el estudio hidráulico se utiliza un modelo bidimensional, concretamente, el modelo GUAD-2D, a partir del cual se obtienen los caudales y las velocidades de los distintos cauces fluviales. En definitiva, se obtienen tres mapas de peligrosidad con la zona inundable y los calados para los períodos de retorno de 10, 100 y 500 años, determinando de esta manera también la probabilidad estadística, siendo alta, media y baja, respectivamente. Para determinar el riesgo existente es necesario conocer la exposición y la vulnerabilidad, a partir de los usos del suelo, de la población y sus bienes. En este sentido, y nuevamente basándose en el Real Decreto 903/2010, se muestra el riesgo para la población, para la actividad económica, para los puntos de especial importancia y para las áreas que se encuentran protegidas medioambientalmente. En el primero se conoce la población estimada que se encuentra dentro de la zona inundable, ya sea según término municipal o distrito censal (Ministerio de Agricultura, Alimentación y Medio Ambiente, 2013).

Por su parte, la Comunidad Valenciana fue pionera en la realización de mapas de riesgo de inundación, respecto al ámbito nacional. En primera instancia, el PATRICOVA de 2003, presentaba una cartografía de peligrosidad que tenía en cuenta los periodos de retorno $(25,100$ y 500 años) y la altura de la lámina de agua (inferior o superior a $0,80 \mathrm{~m}$ ). Con la llegada de la Directiva 2007/60/CE, se replantearon los criterios por los que se rige dicha cartografía. Con ello, en la revisión del PATRICOVA 2015, se incorporan los criterios de vulnerabilidad social y económica para establecer los niveles de riesgo de inundación y se incorporan los criterios geomorfológicos para mejorar la determinación de la peligrosidad de inundación (Olcina Cantos \& Díez-Herrero, 2017; Soriano García et al., 2020). La revisión en 2015, renueva el planteamiento conceptual y metodológico que se había adoptado en el PATRICOVA de 2003. En este primer planteamiento sólo tiene en cuenta el riesgo para medir la frecuencia y magnitud de la inundación, y la vulnerabilidad, para cuantificar la dimensión de los daños causados por inundación.

El planeamiento metodológico de la revisión del PATRICOVA de 2015, redefine los conceptos de peligrosidad y riesgo, distingue los diferentes tipos de inundación e incorpora al concepto de peligrosidad la variable geomorfológica. En cuanto a la vulnerabilidad, se incluyen los aspectos sociales y 
Olcina Cantos, J., Oliva Cañizares, A., Sánchez Almodóvar, E., Martí Talavera, J., Biener Camacho, S. (2021). Cartografías para la acreditación del riesgo de inundaciones: SNCZI y PATRICOVA en la Comunidad Valenciana (España). GeoFocus (Artículos), Revista Internacional de Ciencia y Tecnología de la Información Geográfica, 27, 19-53. http://dx.doi.org/10.21138/GF.691

medioambientales, además esta revisión considera los efectos del cambio climático, tales como los cambios de usos del suelo. En el nuevo planteamiento se sustituye el concepto de riesgo por peligrosidad, ya que es la interrelación de la peligrosidad de inundación y la vulnerabilidad, la que dan lugar al riesgo, denominado en el anterior plan como impacto. La revisión del PATRICOVA toma como base, para la realización de la cartografía de peligrosidad, los antecedentes empleados en el PATRICOVA 2003 y la cartografía modificada durante la vigencia de dicho plan. La elaboración del nuevo mapa de peligrosidad de inundación por criterios geomorfológicos amplía su escala a 1:25 000. La inclusión de la peligrosidad geomorfológica en la revisión del PATRICOVA, ha sido un aspecto novedoso y de vital de importancia, ya que es un elemento fundamental en la prevención del riesgo y la salvaguarda de vidas humanas. Para la consecución de la cartografía de vulnerabilidad se tiene en cuenta los elementos expuestos al riesgo o susceptibles de ser afectados por una inundación (vulnerabilidad económica, social, medioambiental y población afectada). El análisis de la vulnerabilidad pretende cuantificar los daños que produce una inundación. Como regla general se establece que, para cualquier uso, el porcentaje de daños es pequeño cuando el calado es inferior a 70-80 cm y, a partir de este umbral, el valor de los daños aumenta, debido al conocido como "efecto mesa", con el cual, incrementan las pérdidas de forma exponencial, hasta llegar a calados $1,20 \mathrm{~cm}$, a partir del cual comienzan a descender paulatinamente los daños. Algunas de las variables tenidas en cuenta para abordar el análisis de la vulnerabilidad son los cambios de usos del suelo y el incremento de la población y las viviendas. En las últimas décadas ha tomado especial interés el estudio de la vulnerabilidad social ligada a riesgos naturales mediante la creación de índices que analizan las variables socioeconómicas y sociodemográficas (Cutter et al., 2000, 2003; Pérez et al., 2016; Sortino $\&$ Perles, 2017).

A modo de resumen, la siguiente tabla muestra los criterios oficiales que se manejan para la confección de mapas de peligrosidad y de riesgo, según los dos servidores oficiales de cartografía de riesgo.

La determinación de la cartografía de riesgo se basa en tres factores: económicos, sociales y medioambientales afectados por la inundación, este último incluido en la revisión del Plan (Generalitat Valenciana. Consellería d'Habitatge, 2015). Hay que tener en cuenta que los mapas de riesgo se han realizado a través de la cartografía de inundabilidad (peligrosidad 1-6), sin tener en cuenta la peligrosidad geomorfológica que, en muchas ocasiones, es la auténtica causante de inundaciones catastróficas con efectos socioeconómicos muy negativos. Por consiguiente, la cartografía de riesgo del PATRICOVA, no representa el riesgo real existente en toda la Comunidad Valenciana al no incorporarse la peligrosidad geomorfológica en la determinación de los mapas y grados de riesgo.

Para analizar la situación actual de la ubicación de las construcciones en zonas afectadas por la peligrosidad o riesgo de inundación, se han cruzado las capas de información geográfica (en formato (.shp) relativas a la peligrosidad y riesgo de ambos sistemas con las construcciones existentes, que facilita la cartografía del ICV. Asimismo, se ha realizado el geoproceso union en SAGA, para unir en una misma capa shape, todas las construcciones de los municipios que se hallan en la zona de estudio. Posteriormente, se han individualizado las capas de peligrosidad y de riesgo, según sus categorías, para que, mediante un geoproceso de cortado en SAGA denominado Polygon Clipping, obtener el número de edificaciones que se encuentran en cada nivel de peligrosidad y de riesgo de ambas cartografías oficiales 
Olcina Cantos, J., Oliva Cañizares, A., Sánchez Almodóvar, E., Martí Talavera, J., Biener Camacho, S. (2021). Cartografías para la acreditación del riesgo de inundaciones: SNCZI y PATRICOVA en la Comunidad Valenciana (España). GeoFocus (Artículos), Revista Internacional de Ciencia y Tecnología de la Información Geográfica, 27, 19-53. http://dx.doi.org/10.21138/GF.691

Tabla 1. Criterios para la elaboración de los mapas de riesgo de inundación.

\begin{tabular}{|c|c|c|}
\hline CRITERIO & SNCZI & PATRICOVA \\
\hline PELIGROSIDAD & $\begin{array}{l}3 \text { escenarios de probabilidad: } \\
\text { a) Alta, } 10 \text { años periodo de retorno } \\
\text { b) Media, } 100 \text { años período de retorno } \\
\text { c) Baja, } 500 \text { años período de retorno. } \\
\text { Criterios de representación: } \\
\text { a) Hidrológico-Hidráulico } \\
\text { b) Histórico } \\
\text { c) Geomorfológico } \\
\text { Elementos de representación: } \\
\text { a) Extensión de la inundación } \\
\text { b) Calados del agua o nivel del agua } \\
\text { según proceda } \\
\text { c) Velocidad de la corriente o el caudal de } \\
\text { agua correspondiente } \\
\text { Componentes de la legislación de aguas y } \\
\text { RDPH } \\
\text { Determinación de la Zona de Flujo } \\
\text { Preferente } \\
\text { Determinación del Dominio Público } \\
\text { Hidráulico } \\
\text { Selección de zonas: análisis de zonas de } \\
\text { riesgo significativo (ARPSIs), primer y } \\
\text { segundo ciclo de implantación de la } \\
\text { Directiva } 60 / 2007 / C E \text {. }\end{array}$ & $\begin{array}{l}\text {-Peligrosidad hidrológica: } 6 \text { niveles definidos a } \\
\text { partir de dos variables (período de retorno - } \\
\text { 25,100 y } 500 \text { años- y calado }-<80 \mathrm{~cm},>80 \mathrm{~cm}- \\
\text { ). } \\
\text {-Peligrosidad geomorfológica: formas del } \\
\text { territorio que son el resultado de procesos de } \\
\text { arroyada concentrada o difusa. Se han tenido } \\
\text { en cuenta las siguientes: } \\
\text { 1. Cauces. } \\
\text { 2. Barrancos y vaguadas de fondo plano. } \\
\text { 3. Llanura aluvial o llanuras de inundación } \\
\text { (incluye } \\
\text { crestas aluviales y paleocauces). } \\
\text { 4. Abanicos torrenciales. } \\
\text { 5. Abanicos aluviales (incluye paleocauces y } \\
\text { depresiones inter-conos). } \\
\text { 6. Glacis. } \\
\text { 7. Derrames. } \\
\text { 8. Humedales (incluye endorreísmos, } \\
\text { semiendorreísmos, } \\
\text { lagunas y embalses). } \\
\text { 9. Dolinas y poljes. } \\
\text { 10. Restingas. }\end{array}$ \\
\hline RIESGO & $\begin{array}{l}4 \text { elementos de representación: } \\
\text { a) Población (último padrón municipal } \\
\text { disponible) } \\
\text { b) Actividad económica (20 usos del } \\
\text { suelo definidos en el } 1^{\circ} \text { ciclo de } \\
\text { implantación de la Directiva } 60 / 2007 \text {, } \\
\text { asimilables a las } 8 \text { categorías } \\
\text { identificadas por la Comisión Europea) } \\
\text { c) Puntos de especial importancia } \\
\text { (emisiones industriales; EDAR; } \\
\text { patrimonio cultural; elementos } \\
\text { significativos para protección civil; } \\
\text { d) Áreas de importancia ambiental (masas } \\
\text { de agua según Directiva 60/2000; } \\
\text { acuíferos para consumo humano; masas } \\
\text { agua uso recreativo; LICs) }\end{array}$ & $\begin{array}{l}6 \text { elementos de representación para } 3 \text { categorías } \\
\text { principales (carácter económico, social y } \\
\text { medioambiental): } \\
\text { a) La población total del término municipal. } \\
\text { b) El peso de los sectores productivos (según el } \\
\text { empleo total del municipio). } \\
\text { c) La proporción de población activa ligada a la } \\
\text { agricultura. } \\
\text { d) El valor y la composición del parque de } \\
\text { viviendas. } \\
\text { e) El porcentaje de la superficie afectada por la } \\
\text { inundación. } \\
\text { f) La densidad de la población. }\end{array}$ \\
\hline
\end{tabular}

Fte.: SNCZI y el PATRICOVA (2003 y 2015). Elaboración propia. 


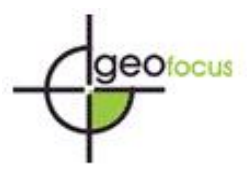

Olcina Cantos, J., Oliva Cañizares, A., Sánchez Almodóvar, E., Martí Talavera, J., Biener Camacho, S. (2021). Cartografías para la acreditación del riesgo de inundaciones: SNCZI y PATRICOVA en la Comunidad Valenciana (España). GeoFocus (Artículos), Revista Internacional de Ciencia y Tecnología de la Información Geográfica, 27, 19-53. http://dx.doi.org/10.21138/GF.691

\section{Análisis y descripción de resultados.}

\subsection{Análisis comparativo sobre la peligrosidad de inundación por las cartografías del SNCZI y el PATRICOVA.}

Del análisis comparativo propuesto para el factor de peligrosidad en el PATRICOVA y las zonas inundables del SNCZI, se observan ciertas diferencias en diversas zonas en el mapa comparativo, donde las mayores diferencias se aprecian en las zonas de peligrosidad geomorfológica que presenta el PATRICOVA y que no son recogidas por el SNCZI.

A grandes rasgos, la mayor parte de la zona de estudio, coincide entre ambas cartografías. Sin embargo, existen otros espacios que contemplan el PATRICOVA pero el SNCZI no. Por ejemplo, las zonas que se hallan al norte de Rafal y al este de Callosa del Segura. Gran parte del término municipal de Catral, y pequeños espacios aislados de diferentes municipios como Orihuela, Jacarilla, Almoradí, Daya Nueva, Daya Vieja, San Fulgencio y Guardamar del Segura. En cambio, las zonas contempladas por el SNCZI que no recoge el PATRICOVA, representa superficies pequeñas que se localizan de manera puntual por la Vega Baja. Sirva de ejemplo, el espacio comprendido al sur de la ciudad de Orihuela, al oeste de la pedanía de Arneva; o sectores de San Felipe Neri o Benijófar (Figura 3).

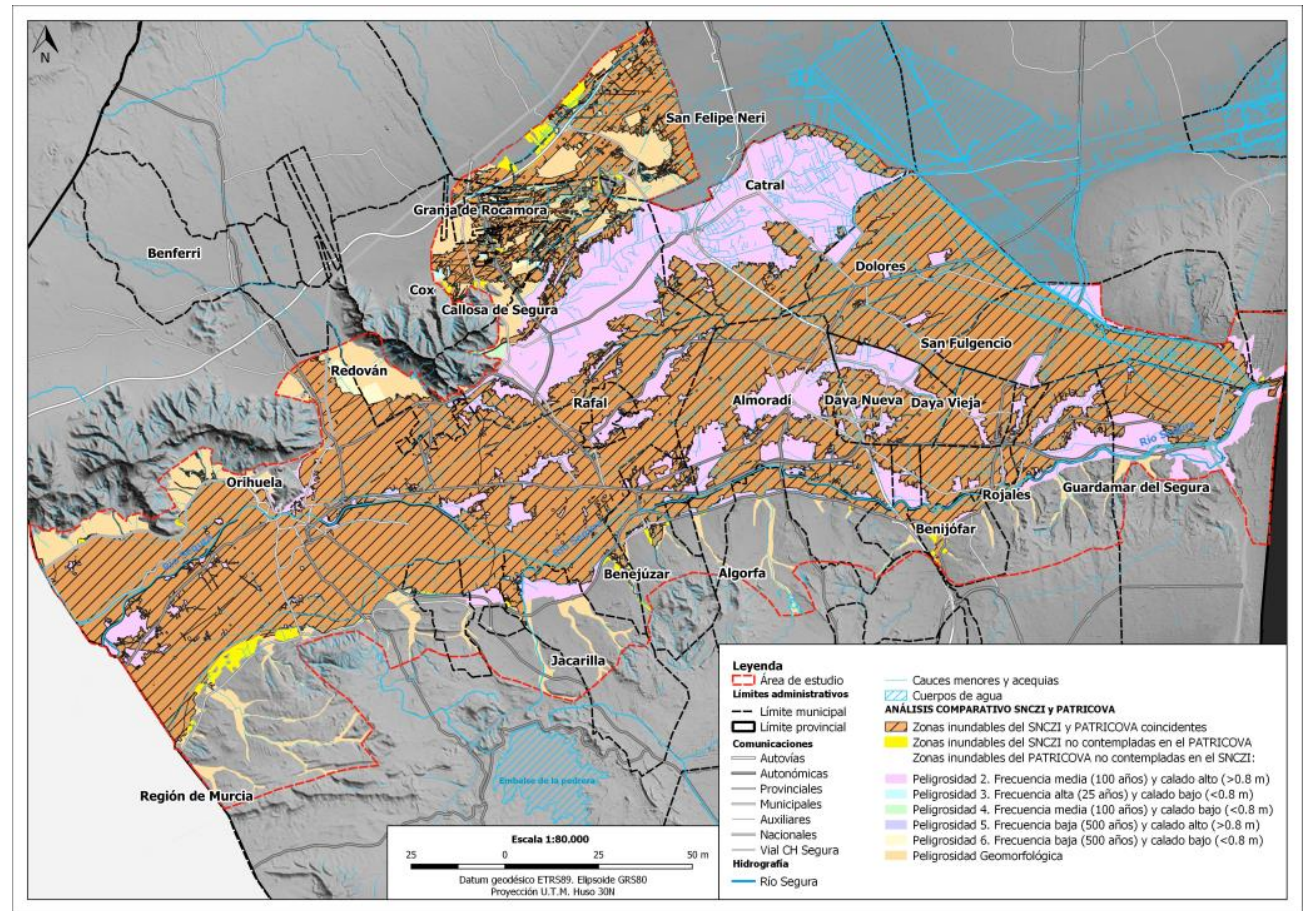

Figura 3. Análisis comparativo para el factor de peligrosidad (PATRICOVA y SNCZI). Fte.: SNCZI y PATRICOVA. Elaboración propia 


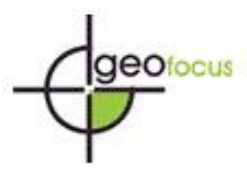

Olcina Cantos, J., Oliva Cañizares, A., Sánchez Almodóvar, E., Martí Talavera, J., Biener Camacho, S. (2021). Cartografías para la acreditación del riesgo de inundaciones: SNCZI y PATRICOVA en la Comunidad Valenciana (España). GeoFocus (Artículos), Revista Internacional de Ciencia y Tecnología de la Información Geográfica, 27, 19-53. http://dx.doi.org/10.21138/GF.691

En efecto, si se realiza una comparativa estadística sobre la peligrosidad y el riesgo de inundación de ambas cartografías, se puede reseñar que de la superficie total que abarca la zona de estudio escogida $\left(302,22 \mathrm{~km}^{2}\right)$, el SNCZI, atendiendo exclusivamente a la peligrosidad por inundación hidrológicahidráulica, viene a representar el $60 \%$ de la superficie total, como zona inundable. Por su parte, el PATRICOVA al incluir el elemento de peligrosidad geomorfológica, el porcentaje de superficie inundable de la zona de estudio escogida, asciende a $80,07 \%$. Esto supone que existe algo más de un $20 \%$ de diferencia, sobre espacios inundables, aproximadamente, entre el SNCZI y el PATRICOVA. A efectos territoriales, esto supone la incorporación o no de construcciones en zonas inundables, lo que supone un incremento del riesgo de inundación en estos espacios donde no existe una cartografía de riesgo que incluya la capa de peligrosidad geomorfológica.

Al unir todas las capas de peligrosidad hidrológica-hidráulica, se obtiene que, prácticamente todo el territorio que conforma la denominada Vega Baja, presenta una peligrosidad de inundación importante, en el que cualquier rincón de dicho espacio, puede verse afectado por las inundaciones. Los únicos espacios que no se ven afectados, son las zonas más elevadas, respecto a la huerta. No obstante, la mayor parte de los piedemontes de los relieves y cabezos, presentan elementos geomorfológicos que actúan como cauces que favorecen o potencian las inundaciones aguas abajo (Figura 4).

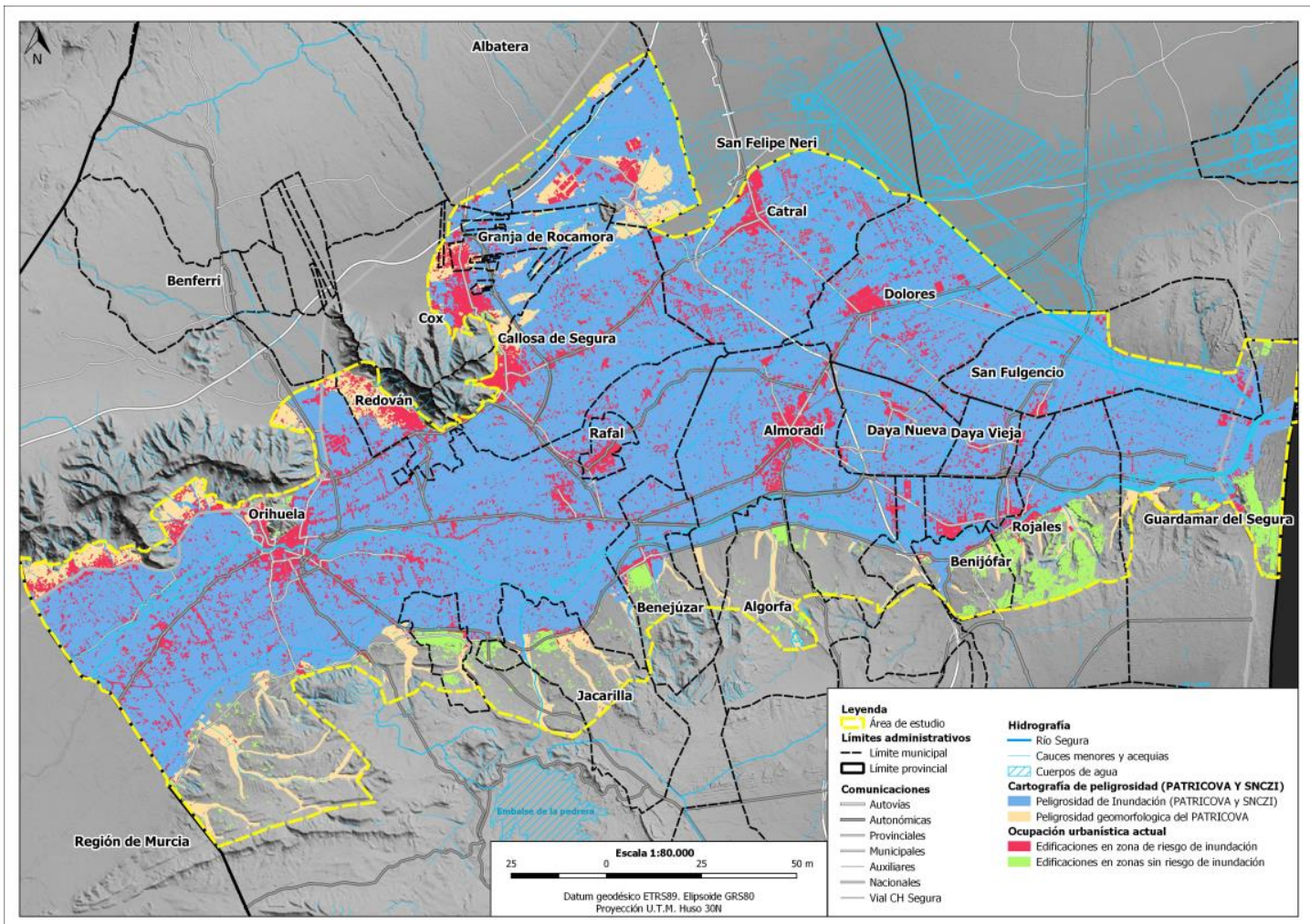

Figura 4. Análisis comparativo para el factor de peligrosidad (PATRICOVA y SNCZI). Fte.: SNCZI y PATRICOVA. Elaboración propia 
Olcina Cantos, J., Oliva Cañizares, A., Sánchez Almodóvar, E., Martí Talavera, J., Biener Camacho, S. (2021). Cartografías para la acreditación del riesgo de inundaciones: SNCZI y PATRICOVA en la Comunidad Valenciana (España). GeoFocus (Artículos), Revista Internacional de Ciencia y Tecnología de la Información Geográfica, 27, 19-53. http://dx.doi.org/10.21138/GF.691

Como se observa en la Figura 4, existen una gran ocupación de construcciones y edificaciones en la Vega Baja, es decir, en una zona inundable. Ello supone que es necesario comprender que existe un riesgo de inundación muy elevado en este sector y que, por consiguiente, no se debe favorecer las construcciones de nuevas infraestructuras, puesto que se estaría incrementando el número de edificaciones (exposición y vulnerabilidad) en dicha zona inundable, lo que se traduce en mayores pérdidas de vidas humanas, ambientales y económicas. Existen construcciones que no ocupan los espacios de peligrosidad hidrológico-hidráulico de ambas cartografías, empero, sí que se ven afectadas por la categoría de peligrosidad que, como se verá más adelante, no se recogen en los mapas de riesgo, lo que supone un número considerable de construcciones que pueden verse afectadas por una inundación y que no están contempladas como zonas de riesgo, vitales para la gestión de la emergencia.

Es por ello que, a pesar de la normativa urbanística que recoge el PATRICOVA, en lo que se refiere a sus artículos 27 y 28, sobre actuaciones de adaptación a las inundaciones en las zonas cuyas edificaciones se encuentran en las categorías de peligrosidad 2,3 y 4, donde la peligrosidad 2 abarca la totalidad de la Vega Baja.

No obstante, estas medidas de adaptación deberían focalizarse en las construcciones existentes y prohibir las nuevas construcciones, lo que supone que los municipios no crezcan ni se desarrollen más de lo que ya están en la actualidad, ya que, como se ha dicho anteriormente, se estaría incrementando el riesgo de estos espacios.

Por estos motivos, se ha elaborado una tabla donde se señala el número de construcciones afectadas (total o parcialmente), según los niveles de peligrosidad del SNCZI y del PATRICOVA, recogida en las siguientes tablas (Tabla 2 y 3 ).

Tabla 2. Superficie inundable y construcciones afectadas según la cartografía de peligrosidad del SNCZI.

\begin{tabular}{|l|c|c|}
\cline { 2 - 3 } \multicolumn{1}{c|}{} & \multicolumn{2}{c|}{ SNCZI (Vega Baja) } \\
\hline P. Muy Alta & Superficie inundable $\left(\mathrm{km}^{2}\right)$ & Construcciones \\
\hline P. Alta & 35,06 & 2.150 \\
\hline P. Media & 63,05 & 4.534 \\
\hline P. Baja & 78,57 & 7.788 \\
\hline
\end{tabular}

Fte.: SNCZI e ICV. Elaboración propia.

Como se observa en la Tabla 3 respecto a la peligrosidad geomorfológica, existe un total de 6.505 construcciones afectadas por zonas inundables, que no se encuentran incorporados en las cartografías de riesgo, lo que supone un auténtico problema en relación a las pérdidas de vidas humanas, económicas y ambientales. Sin embargo, en la actualidad, ni el SNCZI ni el PATRICOVA han incorporado este elemento en la elaboración de los mapas de riesgo. De ahí, la necesidad de reivindicar la incorporación de la peligrosidad geomorfológica a los mapas de riesgo, de manera que configurarían unos resultados más realistas y acordes con la situación "real" de riesgo 


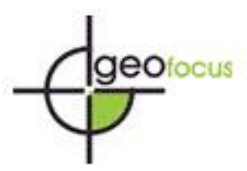

Olcina Cantos, J., Oliva Cañizares, A., Sánchez Almodóvar, E., Martí Talavera, J., Biener Camacho, S. (2021). Cartografías para la acreditación del riesgo de inundaciones: SNCZI y PATRICOVA en la Comunidad Valenciana (España). GeoFocus (Artículos), Revista Internacional de Ciencia y Tecnología de la Información Geográfica, 27, 19-53. http://dx.doi.org/10.21138/GF.691

Tabla 3. Superficie inundable y construcciones afectadas según la cartografía de peligrosidad del PATRICOVA.

\begin{tabular}{|l|c|c|}
\cline { 2 - 3 } \multicolumn{1}{c|}{} & \multicolumn{2}{c|}{ PATRICOVA (Vega Baja) } \\
\hline Peligrosidad 1 & Superficie inundable $\left(\mathrm{km}^{2}\right)$ & Construcciones \\
\hline Peligrosidad 2 & 0,03 & 68 \\
\hline Peligrosidad 3 & 199,20 & 31.848 \\
\hline Peligrosidad 4 & 1,53 & 596 \\
\hline Peligrosidad 5 & 0,17 & 236 \\
\hline Peligrosidad 6 & 0,07 & 142 \\
\hline Peligrosidad Geomorfológica & 5,00 & 527 \\
\hline
\end{tabular}

Fte.: PATRICOVA e ICV. Elaboración propia.

\subsection{Análisis comparativo sobre las cartografías de riesgo de inundación del SNCZI y el PATRICOVA.}

Como se observa en la Figura 5, el riesgo de inundación ocupa la mayor parte del área de estudio, afectando, principalmente, aquellas zonas situadas en la margen izquierda del río Segura, donde la escasa pendiente del terreno contribuye a su anegamiento. Ello supone que no se puede promover nuevas edificaciones en la llanura de inundación de la margen izquierda del río Segura, ni de cualquier otro cauce. De hecho, así lo recogen las cartografías de riesgo que ampara las leyes del suelo vigentes

Por otro lado, es necesario destacar que existen construcciones que no ocupan espacios de riesgo en ambas cartografías, pero, sí que se ven afectadas por la peligrosidad de inundación. Ante esta situación, hay que apelar a la Ley de Suelo y Rehabilitación Urbana de 2015, que dictamina en su artículo 22, que las actuaciones urbanísticas deberán estar avaladas por un informe de sostenibilidad ambiental que incluya un mapa de riesgos naturales (España. Ministerio de Fomento, 2015). En este caso, la Figura 5 muestra el riesgo de inundación presente en la zona, estableciendo las zonas en las que las nuevas construcciones deben evitarse. Por lo tanto, la normativa urbanística del PATRICOVA, en su artículo 16 expone la necesidad de analizar el drenaje superficial del territorio y las cuencas vertiente, teniendo en cuenta la red de drenaje, las zonas de peligrosidad de inundación y las zonas de flujo preferente, mientras que, en su artículo 17 dicta que se clasificará como suelo no urbanizable de especial protección del Dominio Público Hidráulico, así como las zonas de peligrosidad 1 delimitadas por el PATRICOVA, en estas zonas se prohíbe la edificación y en caso de edificarse debe hacerse con unas condiciones especiales, sin dar lugar al incremento del riesgo de inundación. Por consiguiente, las medidas de adaptación al riesgo de inundación deben ir encaminadas a la protección de las construcciones existentes y prohibir los nuevos desarrollos urbanos, sin traspasar los límites de lo ya construido en la actualidad, ya que, se incrementaría la exposición y vulnerabilidad, aumentando el riesgo de estos espacios. 


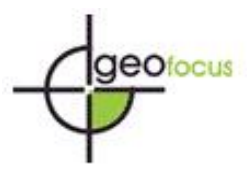

Olcina Cantos, J., Oliva Cañizares, A., Sánchez Almodóvar, E., Martí Talavera, J., Biener Camacho, S. (2021). Cartografías para la acreditación del riesgo de inundaciones: SNCZI y PATRICOVA en la Comunidad Valenciana (España). GeoFocus (Artículos), Revista Internacional de Ciencia y Tecnología de la Información Geográfica, 27, 19-53. http://dx.doi.org/10.21138/GF.691

En cuanto al análisis estadístico referido a la superficie de riesgo contemplada en el área de estudio $\left(302,22 \mathrm{~km}^{2}\right)$, el SNCZI, considerando las zonas de riesgo para la población, el periodo de retorno T500, que es el que presenta una mayor extensión, representa el 61,5\% de la superficie total, como zona de riesgo. Por otro lado, el PATRICOVA, incluyendo sus cuatro niveles de riesgo, representa un 65,06\% de la superficie en la zona de estudio establecida. Ello supone un $4 \%$ de diferencia, aproximada, entre el PATRICOVA y el SNCZI.

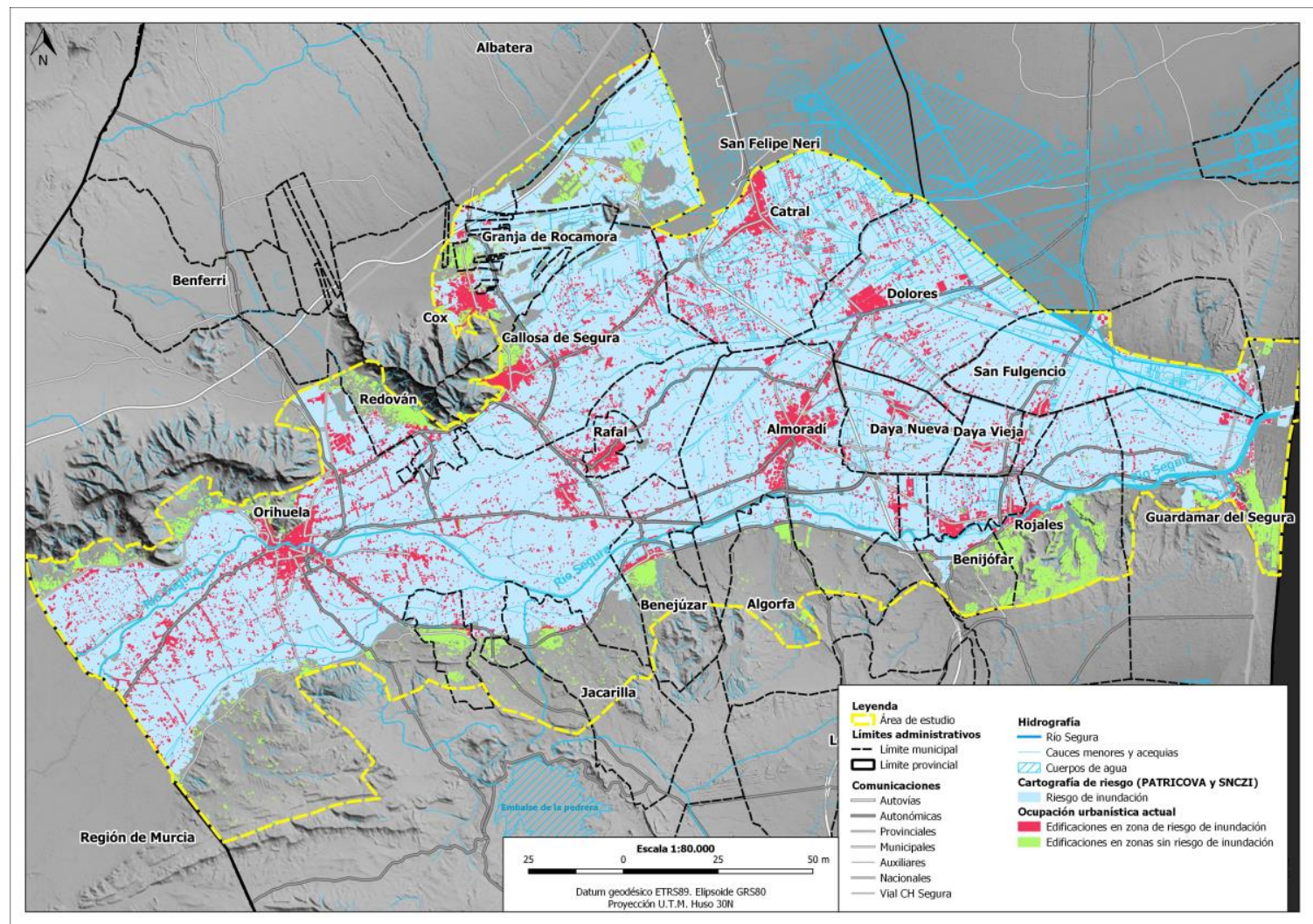

Figura 5. Análisis de las construcciones en zona de riesgo de inundación (PATRICOVA y SNCZI). Fte.: SNCZI y PATRICOVA. Elaboración propia

Como resultado del análisis, se exponen en las siguientes tablas (Tabla 4 y 5), la superficie y las construcciones afectadas por riesgo de inundación según el nivel de riesgo de cada sistema de cartográfico. Destacan las notables diferencias que hay entre las cifras de cada plan, no siendo debido a la distribución de las zonas de riesgo, sino a la metodología utilizada para contabilizar los daños y superficies. En el caso del SNCZI, el riesgo T100 integra a la superficie de T10, más la suya propia, mientras que la superficie de T500 adjunta también a las anteriores junto con la generada ya por ella. Ello impide poder conocer el porcentaje para cada periodo de retorno, ya que, T500 siempre va a suponer el $100 \%$ de la muestra. Por tanto, si no tenemos en cuenta dichas adicciones las superficies para T100 y T500 serían, 41,05 km y 122,07 km², respectivamente. Al trasladar estas cifras a términos porcentuales se observa como T10 sería un 12,2\%, T100 el 22,1\% y, por último, T500 supondría el 65,7\% de la 


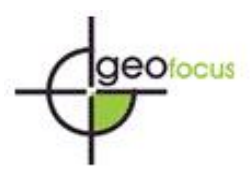

Olcina Cantos, J., Oliva Cañizares, A., Sánchez Almodóvar, E., Martí Talavera, J., Biener Camacho, S. (2021). Cartografías para la acreditación del riesgo de inundaciones: SNCZI y PATRICOVA en la Comunidad Valenciana (España). GeoFocus (Artículos), Revista Internacional de Ciencia y Tecnología de la Información Geográfica, 27, 19-53. http://dx.doi.org/10.21138/GF.691

superficie. Este mismo análisis se puede realizar para las construcciones, donde T10 seguiría teniendo una cifra parecida con un $12,4 \%$, y habría un aumento importante para T100 con respecto a las construcciones, con un $32 \%$, lo que hace que T500 se reduzca hasta el 56,8 \%.

La metodología del PATRICOVA, es distinta, ya que no tiene en cuenta la extensión de la superficie del riesgo inferior, lo que permite trasladar directamente a porcentajes los resultados extraídos. Con ello se extrae que las zonas con un riesgo muy bajo son el 80,24\% de la muestra, seguida de las áreas con un riesgo medio, que serían el 8,63\%, mientras que el resto de categorías no supera el $5 \%$. Cifras totalmente distintas a las calculadas en el caso de las construcciones, ya que en este caso el mayor número de inmuebles permanecen en un área con un riesgo alto y medio, con unos porcentajes que se hallan en $31,37 \%$ y $28,63 \%$, respectivamente.

Tabla 4. Superficie de riesgo y construcciones afectadas según la cartografía de riesgo para la población del SNCZI.

\begin{tabular}{|l|c|c|}
\cline { 2 - 3 } \multicolumn{1}{c|}{} & \multicolumn{2}{c|}{ SNCZI (Vega Baja) } \\
\hline Riesgo T10 & Superficie de riesgo $\left(\mathrm{km}^{2}\right)$ & Construcciones \\
\hline Riesgo T100 & 22,69 & 2.535 \\
\hline Riesgo T500 & 63,74 & 9.098 \\
\hline
\end{tabular}

Fte.: SNCZI e ICV. Elaboración propia.

Algunos de los municipios más poblados de la comarca presentan un importante riesgo, destacando Orihuela, la capital comarcal, y Almoradí y Callosa, con una población que ronda los 20000 habitantes. Además, se observa que una gran cantidad de vivienda dispersa asentada en la huerta y algunas partidas rurales se encuentran en zonas que presentan riesgo de inundación.

Además, en el área de estudio podemos encontrar casos concretos de urbanizaciones, partidas o infraestructuras situadas en sectores propicios a los anegamientos, al menos para el período de retorno de T500, sin olvidar que la cartografía del SNCZI no tiene en cuenta factores asociados a la peligrosidad geomorfológica, en las que se incluyen abanicos aluviales, ramblizos, torrenteras o pequeños sectores endorreicos de fondo plano.

Precisamente, resulta muy llamativo el caso de algunas de las pedanías de Orihuela que se encuentran al pie de la vertiente meridional de la sierra. Destacan los Raigueros de Bonaza, de Levante y La Aparecida, que han crecido sobre los abanicos aluviales de las torrenteras que descienden de la sierra, y que en eventos extremos como el de septiembre de 2019 recuperan su funcionalidad. También se encuentra en esta situación la urbanización del Pinar de Bonanza o en el Rincón de la Seca. En el mismo núcleo urbano de Orihuela, y en concreto en el barrio de Ciudad Jardín, se repite el problema. Son sectores que presentan problemas de anegamientos esporádicos y puntuales en caso de episodios importantes de lluvias de alta intensidad horaria, pero que no aparecen reflejados en la cartografía del SNCZI. En la vertiente septentrional de la sierra de Orihuela se encuentra la urbanización de Montepinar, en un sector de derrame de las ramblas que bajan desde los puntos más elevados de la sierra. En la pedanía de Arneva, unos kilómetros al sur de la capital comarcal y situada al pie del Cabezo de Hurchillo, también se asienta 


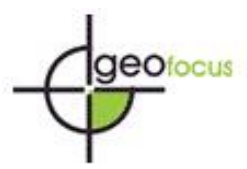

Olcina Cantos, J., Oliva Cañizares, A., Sánchez Almodóvar, E., Martí Talavera, J., Biener Camacho, S. (2021). Cartografías para la acreditación del riesgo de inundaciones: SNCZI y PATRICOVA en la Comunidad Valenciana (España). GeoFocus (Artículos), Revista Internacional de Ciencia y Tecnología de la Información Geográfica, 27, 19-53. http://dx.doi.org/10.21138/GF.691

en un sector con cierta peligrosidad geomorfológica, ya que además en el centro de esta partida confluyen los barrancos procedentes del Cabezo.

Tabla 5. Superficie de riesgo y construcciones afectadas según la cartografía de riesgo del PATRICOVA.

\begin{tabular}{|c|c|c|c|c|}
\cline { 2 - 5 } \multicolumn{1}{c|}{} & \multicolumn{4}{c|}{ PATRICOVA (Vega Baja) } \\
\cline { 2 - 5 } \multicolumn{1}{c|}{} & Superficie riesgo $\left(\mathrm{km}^{2}\right)$ & Porcentaje (\%) & Construcciones & Porcentaje (\%) \\
\hline $\begin{array}{c}\text { Riesgo } \\
\text { Muy Alto }\end{array}$ & 7,48 & 3,80 & 6.003 & 17,34 \\
\hline $\begin{array}{c}\text { Riesgo } \\
\text { Alto }\end{array}$ & 4,90 & 2,49 & 10.861 & 31,37 \\
\hline $\begin{array}{c}\text { Riesgo } \\
\text { Medio }\end{array}$ & 16,97 & 8,63 & 9.914 & 28,63 \\
\hline $\begin{array}{c}\text { Riesgo } \\
\text { Bajo }\end{array}$ & 9,51 & 4,84 & 3.199 & 13,42 \\
\hline $\begin{array}{c}\text { Riesgo } \\
\text { Muy } \\
\text { Bajo }\end{array}$ & 157,77 & 80,24 & 4.646 & 9,24 \\
\hline
\end{tabular}

Fte.: PATRICOVA e ICV. Elaboración propia.

Los núcleos urbanos de Callosa y Redován se encuentran en la misma situación, y como se puso de manifiesto durante las inundaciones de septiembre de 2019, sufren anegamientos de forma periódica. En el caso de Callosa hay un largo historial de daños en el centro del municipio por avalanchas de agua y lodo que descienden de los barrancos de la sierra.

Por otra parte, existe la presencia de polígonos industriales, centros comerciales $\mathrm{u}$ otras infraestructuras ya construidas o previstas que se asientan en la vega o en sectores endorreicos que presentan un elevado riesgo. En el entorno de Almoradí se encuentran varios ejemplos de ello. Destaca el polígono industrial de Las Maromas, muy cerca del cauce del Segura, que sufrió graves inundaciones en septiembre de 2019 por el desbordamiento del río y de las acequias, a lo que hay que sumar la rotura de las motas. No hay que olvidar que buena parte del término municipal de esta localidad se encuentra a una altitud inferior a la de las motas, por lo que resulta muy complicada la evacuación de las aguas. Está proyectada la ampliación del polígono en un futuro. Otro de los proyectos futuros es el del centro comercial, que se situaría al igual que el polígono en una zona de riesgo según la cartografía del PATRICOVA y del SNCZI. En el proyecto del nuevo PGOU se recogía la construcción de una zona residencial y un centro comercial en la carretera que conecta Almoradí con la pedanía El Saladar y Rafal. Todo este sector acabó bajo las aguas en las inundaciones de 2019, por el desbordamiento del azarbe Mayayo. Precisamente, la pedanía almoradidense de El Saladar suele ser una de las más afectadas en la comarca por los anegamientos durante episodios de precipitaciones intensas.

Otro de los polígonos de la comarca afectados por probables inundaciones es el de Puente Alto, cerca de la pedanía oriolana de El Escorratel, en un sector que presenta riesgo de inundación según los 


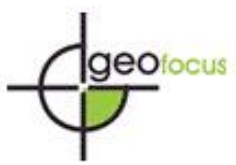

Olcina Cantos, J., Oliva Cañizares, A., Sánchez Almodóvar, E., Martí Talavera, J., Biener Camacho, S. (2021). Cartografías para la acreditación del riesgo de inundaciones: SNCZI y PATRICOVA en la Comunidad Valenciana (España). GeoFocus (Artículos), Revista Internacional de Ciencia y Tecnología de la Información Geográfica, 27, 19-53. http://dx.doi.org/10.21138/GF.691

mapas del PATRICOVA y el SNCZI. Aunque hoy en día no sea apreciable, la rambla de Abanilla, conocida aguas arriba como río Chícamo, discurría en lo que hoy en día son viviendas y campos de cultivo, desaguando al Segura en las cercanías de la partida de La Campaneta. En las inundaciones de 2019 , los extraordinarios caudales y anegamientos provocados por la rambla es un importante toque de atención, ya que muchas personas desconocen la peligrosidad de este aparato fluvial. Muy cerca de Puente Alto, está prevista la construcción del nuevo Parque de Bomberos de Orihuela, en una parcela situada cerca de la urbanización de Montepinar, en el cruce de la carretera de La Murada hacia La Matanza. Se trata de una zona con riesgo muy alto al estar contigua a la rambla de Abanilla, a lo que hay que sumar que se encuentra en una zona de derrames y abanicos de las ramblas que descienden de la Sierra de Orihuela, como se ha explicado en las líneas anteriores.

Otro buen ejemplo de esta problemática se localiza al norte del casco urbano de Redován, situado en un sector de peligrosidad geomorfológica según el PATRICOVA, por las torrenteras de las montañas y los abanicos aluviales que allí se encuentran, muy cerca también de la rambla de Abanilla. En concreto, una pequeña zona industrial, el cementerio y el polígono de San Carlos, en el extremo septentrional del área de estudio se encuentran dentro de un área establecida de peligro geomorfológico por el PATRICOVA.

Por último, el polígono del Mos del Bou, entre los términos de Albatera, Cox y de la Granja de Rocamora se sitúa también en una zona con riesgo de inundación.

Por tanto, a la vista de los recientes acontecimientos y en el contexto actual de cambio climático, con eventos de precipitaciones cada vez más extremos, resulta muy aconsejable tener en cuenta el factor de la peligrosidad geomorfológica del PATRICOVA para corregir o mitigar las consecuencias de avenidas extraordinarias como las de septiembre de 2019.

\section{Discusión y valoración de hallazgos}

La existencia de dos cartografías oficiales de inundación en la Comunidad Valenciana genera la duda de la prevalencia de una sobre otra a efectos de su manejo en los procesos de ordenación territorial. El artículo 7 de la normativa del PATRICOVA se establece que la relación entre las cartografías de peligrosidad y riesgo de inundación elaborada por las Demarcaciones Hidrográficas y por la Generalitat tendrá carácter complementario, aunque las metodologías de trabajo hayan sido diferentes. Es por ello que la elaboración de una única cartografía, como fusión de las dos existentes, no ha sido posible. La Generalitat Valenciana, en su "Guía de aplicación" del PATRICOVA (2019) señala que la cartografía de la Generalitat será complementaria de la elaborada por el SNCZI en aquellos lugares donde no se hayan elaborado mapas de peligrosidad y riesgos por parte de la Demarcación Hidrográfica correspondiente. Por tanto, la cartografía a consultar en primera aproximación es la del SNCZI y si esta no está realizada para el ámbito de planificación, se manejará la del PATRICOVA (art. 7, Normativa, PATRICOVA).

Conviene recordar, sin embargo, que la cartografía del SNCZI afecta tan sólo a las denominadas Áreas de Riesgo Potencial Significativo de Inundación (ARPSIs) que, a final del $1^{\circ}$ ciclo de implantación del SNCZI afecta, tan sólo al $10 \%$ de la superficie total de la Comunidad Valenciana. De manera que ambas cartografías de inundación deben tener un manejo constante para evitar la ocupación con usos no permitidos de áreas con riesgo. 


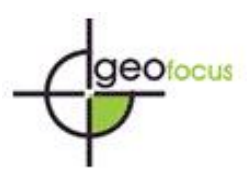

Olcina Cantos, J., Oliva Cañizares, A., Sánchez Almodóvar, E., Martí Talavera, J., Biener Camacho, S. (2021). Cartografías para la acreditación del riesgo de inundaciones: SNCZI y PATRICOVA en la Comunidad Valenciana (España). GeoFocus (Artículos), Revista Internacional de Ciencia y Tecnología de la Información Geográfica, 27, 19-53. http://dx.doi.org/10.21138/GF.691

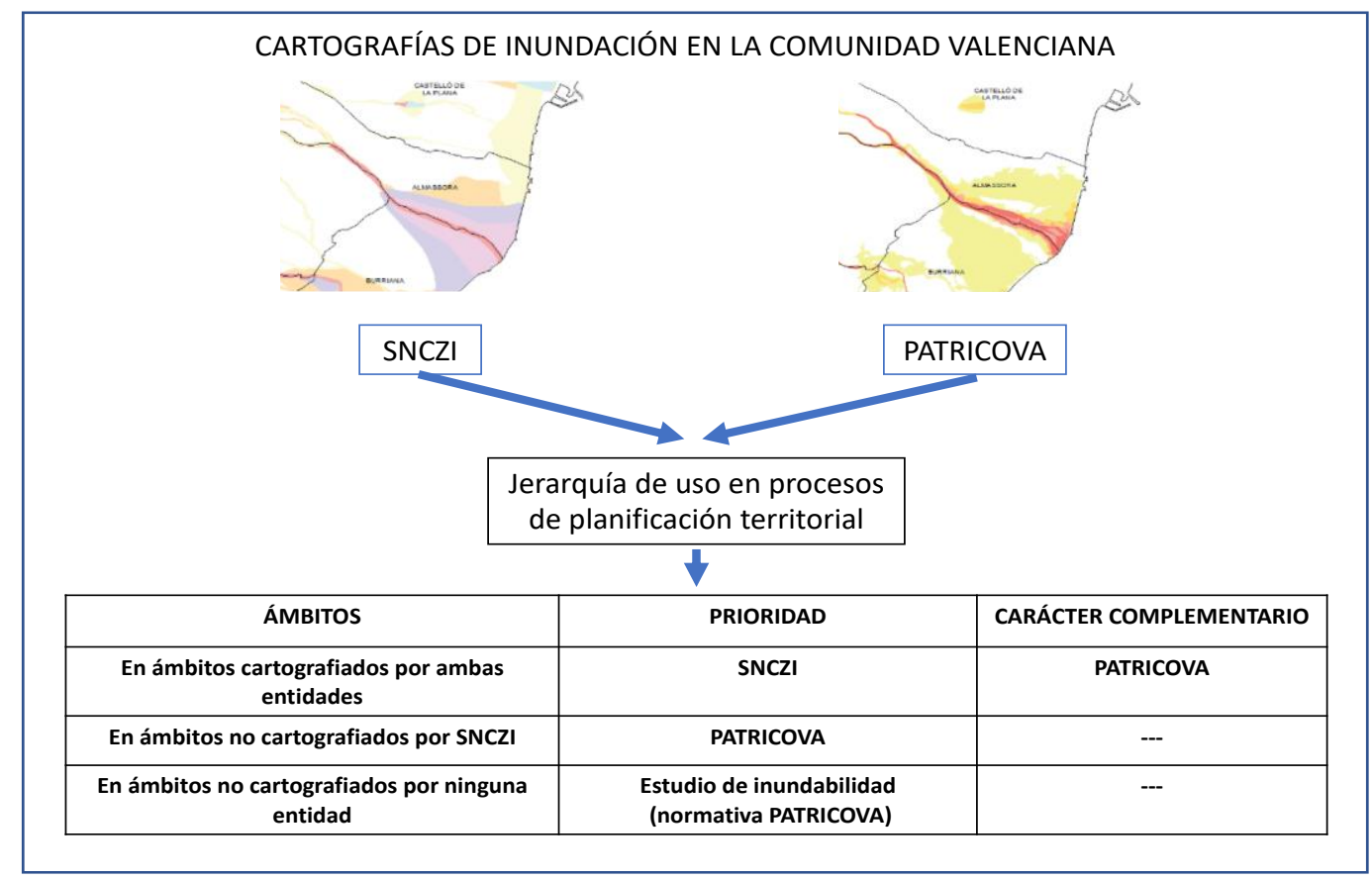

Figura 6. Jerarquía oficial de uso de las cartografías de inundación en la Comunidad Valenciana.

Fte.: Elaboración propia.

En el caso de que haya contradicción, por diferencia de criterio, en los mapas de ambas entidades prevalece la cartografía del SNCZI en aquellos ámbitos que hayan sido abordados por ambos (art.10.3. Normativa, PATRICOVA). Si el área de estudio o planificación no ha sido cartografiada por el SNCZI, prevalece la cartografía del PATRICOVA.

Por último, si un tramo de curso fluvial no ha sido cartografiado ni por el SNCZI ni por el PATRICOVA, y pueda ser afectado por un proceso de planificación urbanística o territorial, se aplicará lo contenido en el art. 12 del PATRICOVA relativo a la elaboración de estudio de inundabilidad con arreglo a los criterios del plan regional.

Todo ello implica que la cartografía es la herramienta básica para la acreditación del riesgo y que, para consultar la peligrosidad o el riesgo de inundación de un territorio para cualquier finalidad en materia de ordenación del territorio y planificación urbanística, en primera instancia, debe consultarse el SNCZI y, seguidamente, el PATRICOVA, para completar de la manera más ajustada posible, la peligrosidad y riesgo existente en la Comunidad Valenciana.

Un aspecto a tener en cuenta y considerar en la revisión de las cartografías, es incluir un criterio novedoso basado en el riesgo de las vidas humanas. Esto supone un cambio de paradigma, pero complementaria al existente. Con el análisis de la metodología empleada por el SNCZI y el PATRICOVA, se observa que los criterios de peligrosidad y calado están basados en periodos de retorno y, con un valor a modo de umbral, donde calados inferiores a $0.80 \mathrm{~m}$, genera daños económicos menos importantes, que los 


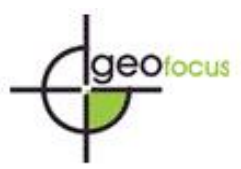

Olcina Cantos, J., Oliva Cañizares, A., Sánchez Almodóvar, E., Martí Talavera, J., Biener Camacho, S. (2021). Cartografías para la acreditación del riesgo de inundaciones: SNCZI y PATRICOVA en la Comunidad Valenciana (España). GeoFocus (Artículos), Revista Internacional de Ciencia y Tecnología de la Información Geográfica, 27, 19-53. http://dx.doi.org/10.21138/GF.691

que superan dicho valor, donde los daños económicos crecen de manera exponencial. Este criterio está basado en el denominado <<efecto mesa〉>.

Sin embargo, se trata de un calado muy elevado que para nada corresponde con la realidad de un episodio de inundación para el riesgo de las vidas humanas, por lo que en determinados episodios de inundación, algunas personas terminan perdiendo su vida, bien por ahogamiento por inundación o por arrastre de las aguas, por ejemplo: caer en una acequia de riego azarbe o por la fuerza de las aguas desbordadas de un río, cauce intermitente, o de la propia morfología de las calles con pendientes pronunciadas que correspondían a antiguos barrancos o ramblas (incorporados en la trama urbana), y que recuperan su función natural, o bien las propias calles que actúan como éstos.

A partir de estas afirmaciones, se hace necesario establecer una metodología basada en una serie de intervalos o umbrales que, en función del mayor o menor calado, junto con la velocidad de las aguas (fuerza), y relacionado con la integridad física de una persona; se pueda delimitar unos niveles de riesgo más acorde con la realidad de un episodio de inundación en un espacio muy ocupado por la población, como sería el caso de la Vega Baja del Segura, entre otros tantos espacios del territorio español. Asimismo, esta cartografía serviría de manera complementaria a la existente basada en criterios económicos.

Por ello, se propone la siguiente clasificación en función del peligro que supone a una persona humana que, posteriormente definiría un mapa de riesgo. Como se observa en la Tabla 6, el calado se ha ajustado a intervalos que pueden afectar a las personas, sin olvidar, la importancia de la velocidad de las aguas. Atendiendo a este último aspecto, el valor de las aguas asignado es el establecido por el RD 9/2008 de modificación del RDPH. Solventado el problema que pudiera suponer esta cuestión, conviene matizar en más detalle los intervalos de calado establecidos en función de la integridad física de las personas.

- Calado 0-0.20 m: Este primer grupo corresponde con los primeros instantes de precipitación, y generación de escorrentía superficial. Se trata de un calado ínfimo que alcanza la altura de los pies y tobillos de las personas, capaz de arrastrarlas si la velocidad de las aguas es superior a $1 \mathrm{~m} / \mathrm{s}$.

- Calado 0.20-0.50 m: Este segundo grupo corresponde a la prolongación y perduración de precipitaciones que, de manera constante, comienza a incrementar el calado de la escorrentía superficial. En este intervalo, en función de si la velocidad de las aguas es menor o mayor a $1 \mathrm{~m} / \mathrm{s}$, ya es capaz de arrastrar mobiliario urbano (contenedores, vehículos...), que pueden impactar en personas pudiendo ser arrastradas por la corriente.

- Calado 0.50-0.80 m: Este tercer grupo corresponde a calados importantes dado la altura que representan respecto al suelo. En este intervalo, además de la capacidad de arrastre de personas, en función de si la velocidad de las aguas es menor o mayor a $1 \mathrm{~m} / \mathrm{s}$, puede arrastrar mobiliario urbano de mayor envergadura (contenedores, vehículos, árboles, señales...) y la fuerza de las aguas puede desplazar de vehículos y obstáculos que se encuentre por el camino.

- Calado 0.80-1.20 m: En este intervalo, además de la capacidad de arrastre de personas, es capaz de arrastrar mobiliario urbano, vehículos y cualquier obstáculo que encuentre por el camino.

- Calado > $1.20 \mathrm{~m}$ : Se trata de calados muy peligrosos para las vidas humanas, debido a la capacidad de arrastre de personas unido al arrastre de mobiliario urbano y vehículos pesados que pueden impactar en personas. Esto supone la peligrosidad más elevada ya que puede arrastrar a vehículos con personas dentro. 
Olcina Cantos, J., Oliva Cañizares, A., Sánchez Almodóvar, E., Martí Talavera, J., Biener Camacho, S. (2021). Cartografías para la acreditación del riesgo de inundaciones: SNCZI y PATRICOVA en la Comunidad Valenciana (España). GeoFocus (Artículos), Revista Internacional de Ciencia y Tecnología de la Información Geográfica, 27, 19-53. http://dx.doi.org/10.21138/GF.691

El resultado final de los mapas de riesgo de inundación que se elaboren por medio de esta metodología, mostraría resultados bastante diferentes a los acostumbrados por el SNCZI y el PATRICOVA en la comunidad autónoma valenciana. Además, al tratarse de una clasificación de calados al que se le asocia una peligrosidad, y no de manera viceversa, incorporaría la peligrosidad por inundación y geomorfológica. Asimismo, en esta clasificación se ha tenido en cuenta, episodios de inundación que, por sus características, como el calado y velocidad de las aguas circulantes, han provocado la pérdida de vidas humanas.

Tabla 6. Propuesta metodológica para la elaboración de cartografía de peligrosidad y de riesgo de inundación en función de las vidas humanas

\begin{tabular}{|c|c|c|c|}
\hline Calado (m) & Velocidad (m/s) & Peligrosidad & Descripción \\
\hline \multirow[t]{2}{*}{$0-0.20 \mathrm{~m}$} & $<1 \mathrm{~m} / \mathrm{s}$ & Baja & Escasa capacidad de arrastre \\
\hline & $>1 \mathrm{~m} / \mathrm{s}$ & Media & Alta capacidad de arrastre \\
\hline \multirow[b]{2}{*}{$0.20-0.50 \mathrm{~m}$} & $<1 \mathrm{~m} / \mathrm{s}$ & Media & $\begin{array}{l}\text { Escasa capacidad de arrastre pero calado } \\
\text { considerable para el riesgo de vidas humanas }\end{array}$ \\
\hline & $>1 \mathrm{~m} / \mathrm{s}$ & Media-Alta & $\begin{array}{l}\text { Calado capaz de arrastrar: } \\
\text { a) Personas } \\
\text { b) Contenedores } \\
\text { c) Vehículos } \\
\text { d) Otros }\end{array}$ \\
\hline \multirow[t]{2}{*}{$0.5-0.8 \mathrm{~m}$} & $<1 \mathrm{~m} / \mathrm{s}$ & Alta & $\begin{array}{l}\text { Calado capaz de arrastrar: } \\
\text { a) Personas } \\
\text { b) Contenedores } \\
\text { c) Vehículos } \\
\text { d) Otros }\end{array}$ \\
\hline & $>1 \mathrm{~m} / \mathrm{s}$ & Alta-Muy Alta & $\begin{array}{l}\text { Calado capaz de arrastrar: } \\
\text { a) Personas } \\
\text { b) Contenedores } \\
\text { c) Vehículos } \\
\text { d) Otros }\end{array}$ \\
\hline \multirow{2}{*}{$0.8-1.2 \mathrm{~m}$} & $<1 \mathrm{~m} / \mathrm{s}$ & Muy Alta & $\begin{array}{l}\text { Calado capaz de arrastrar: } \\
\text { e) Personas } \\
\text { f) Contenedores } \\
\text { g) Vehículos } \\
\text { a) Otros }\end{array}$ \\
\hline & $>1 \mathrm{~m} / \mathrm{s}$ & Muy Alta-Extrema & $\begin{array}{l}\text { Calado capaz de arrastrar: } \\
\text { a) Personas } \\
\text { b) Contenedores } \\
\text { c) Vehículos } \\
\text { d) Otros }\end{array}$ \\
\hline \multirow{2}{*}{$+1.2 \mathrm{~m}$} & $<1 \mathrm{~m} / \mathrm{s}$ & Extrema & $\begin{array}{l}\text { Calado capaz de arrastrar: } \\
\text { a) Personas } \\
\text { b) Contenedores } \\
\text { c) Vehículos pesados } \\
\text { d) Otros } \\
\end{array}$ \\
\hline & $>1 \mathrm{~m} / \mathrm{s}$ & Muy Extrema & $\begin{array}{l}\text { Calado capaz de arrastrar: } \\
\text { a) Personas } \\
\text { b) Contenedores } \\
\text { c) Vehículos pesados } \\
\text { d) Otros }\end{array}$ \\
\hline
\end{tabular}

Fte.: Elaboración propia. 
Olcina Cantos, J., Oliva Cañizares, A., Sánchez Almodóvar, E., Martí Talavera, J., Biener Camacho, S. (2021). Cartografías para la acreditación del riesgo de inundaciones: SNCZI y PATRICOVA en la Comunidad Valenciana (España). GeoFocus (Artículos), Revista Internacional de Ciencia y Tecnología de la Información Geográfica, 27, 19-53. http://dx.doi.org/10.21138/GF.691

A modo de ejemplo se ha elaborado un mapa de peligrosidad aplicando la metodología propuesta según el calado y la velocidad de las aguas, en función de la integridad física de las personas. En este sentido, se ha escogido como ejemplo la rambla del Pilar localizada en el núcleo urbano de Callosa del Segura. La elección de dicho municipio corresponde por el ejemplo más evidente de la alternancia de zonas de calados bajos con fuertes velocidades, en lo correspondiente a la rambla alta y rambla baja (rambla del Pilar); y viceversa, calados elevados con velocidades escasas o nulas, en la huerta de Callosa del Segura. Además, de la existencia de videos en la red que ha permitido comparar y contrastar los resultados obtenidos con la realidad del momento, en el episodio de inundación de septiembre de 2019. Los resultados obtenidos de reflejan en el siguiente mapa (Figura 7):

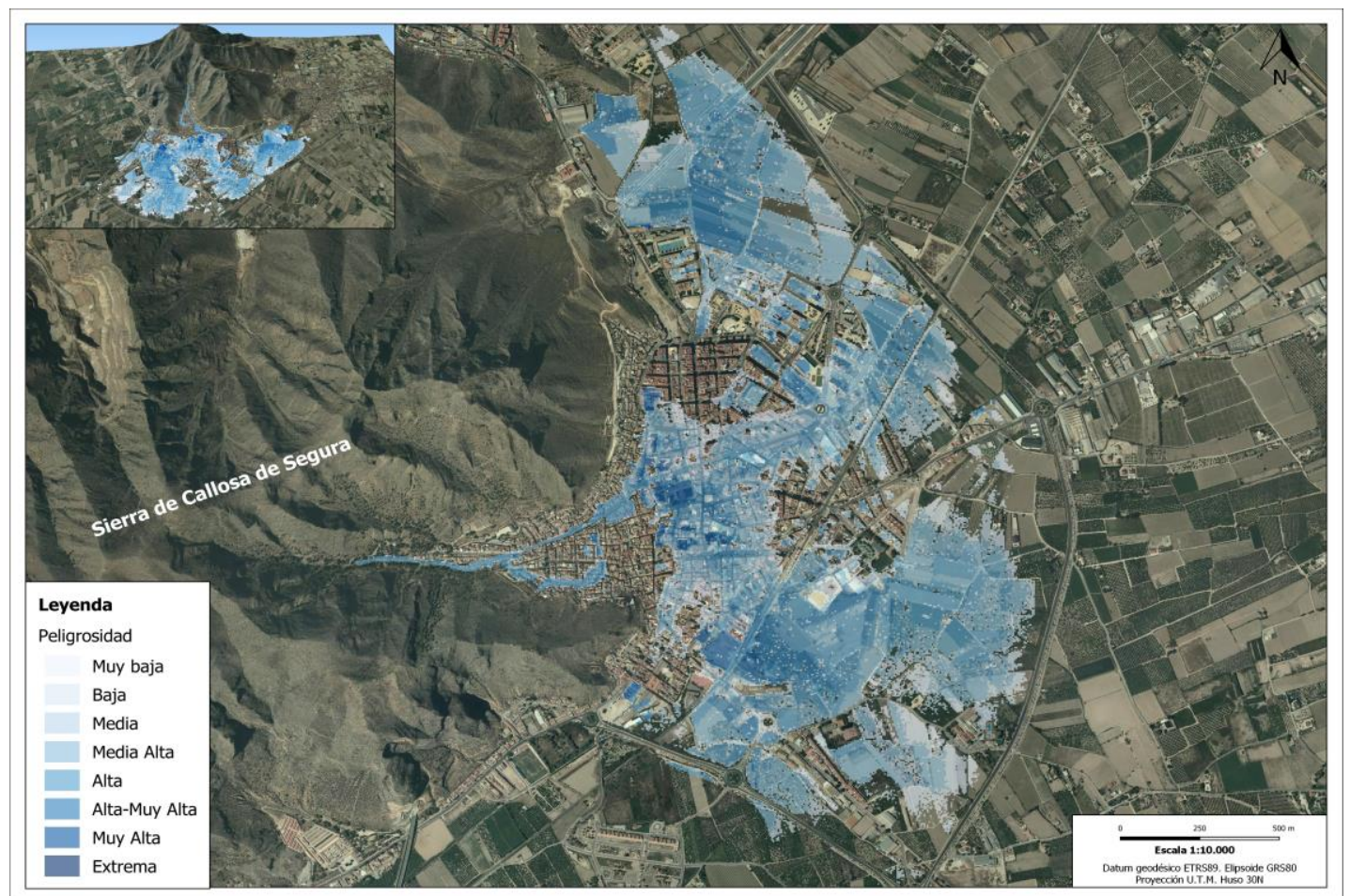

\section{Figura 7. Mapa de peligrosidad según la propuesta metodológica entre la relación de calados, velocidad e integridad física de las personas.}

Fte.: Resultados obtenidos a través de la modelización hidráulica en HEC-RAS 5.0.6. con un caudal de $150 \mathrm{~m}^{3} / \mathrm{s}$. Elaboración propia

La modelización empleada se ha basado en utilizar la rambla del Pilar, cuyo punto de partida es la última presa de contención de sedimentos, su prolongación por la calle rambla del Pilar (rambla alta) y la calle rambla baja, hasta la huerta del municipio. Se ha introducido un caudal de $150 \mathrm{~m}^{3} / \mathrm{s}$, con un régimen crítico, dada enorme pendiente que se halla en este espacio geográfico, correspondiente a un cono aluvial. Una vez realizada la modelización y obtenidas las zonas inundables según su calado y velocidad, se ha procedido a realizar una corrección de la modelización en el tramo alto, reduciendo el calado a valores entre 0.20-0.50 m y/o 0.50-0.80 m, según la metodología propuesta, dado que son valores más reales a los 
Olcina Cantos, J., Oliva Cañizares, A., Sánchez Almodóvar, E., Martí Talavera, J., Biener Camacho, S. (2021). Cartografías para la acreditación del riesgo de inundaciones: SNCZI y PATRICOVA en la Comunidad Valenciana (España). GeoFocus (Artículos), Revista Internacional de Ciencia y Tecnología de la Información Geográfica, 27, 19-53. http://dx.doi.org/10.21138/GF.691

calados producidos. Tras esta corrección se realiza una reclasificación según la tabla 6 sobre calados y velocidades, asignándoles un valor de peligrosidad. La suma de los valores de peligrosidad entre calados y velocidades ha dado como resultado el mapa de peligrosidad, donde se aprecia una peligrosidad elevada en el tramo alto, medio y bajo de la calle rambla, sobre todo por la velocidad de las aguas superiores a $1 \mathrm{~m} / \mathrm{s}(3-5 \mathrm{~m} / \mathrm{s})$; y, a partir de la zona más baja del abanico y de la huerta, la peligrosidad incrementa por los calados acumulados junto a velocidades escasas o nulas. Asimismo, se observa que las zonas inundadas sería el $80 \%$ del núcleo urbano en el tramo bajo, y las zonas de huerta norte, este y sur. Resultados que se asemejan a la inundación de septiembre de 2019 (Figura 7).

A ello, faltaría añadirle el resto de barrancos, torrentes y ramblas que descienden por la sierra de Callosa del Segura hacia el núcleo urbano, como el barranco de Tatus, entre muchos otros; cuyas aportaciones de agua podrían incrementar el caudal y la velocidad de las mismas, modificando la peligrosidad. Además, de que se incorporarían nuevos espacios inundados, en el término municipal de Callosa del Segura, sin contar con un desbordamiento del río Segura.

No obstante, cabe destacar que se podrían incorporar otra serie de elementos que detallen más específicamente zonas de mayor o menos peligrosidad en base a factores físicos (pendiente, sedimentos, derrubios, etc.); y factores humanos (impermeabilidad del suelo, la presencia de vehículos estacionados o en circulación, contenedores, elementos del mobiliario urbano, pasos de agua bloqueados, insuficiencia y/o roturas de la red de saneamiento, etc.). La incorporación de estos elementos, entre otros, podría dar como resultado un mapa de peligrosidad real para la integridad física de las personas.

Si se realiza una comparación entre los resultados obtenidos con la cartografía del SNCZI y el PATRICOVA, los resultados obtenidos son mucho más precisos. Mientras que el SNCZI señala la anegación de la rambla del Pilar (rambla alta y baja) y parte la huerta en la misma trayectoria; el resultado del mapa de peligrosidad con un Q150 m³/s, incluye este sector y otros espacios del núcleo urbano y de la huerta que no contempla el SNCZI.

Este hecho se debe a que, según el documento de Mapas de peligrosidad y riesgo de inundación del SNCZI ( $2^{\circ}$ Ciclo), tras la inundación de noviembre de 1987, se construyeron un sistema de presas de contención de sedimentos y canalizaron el tramo de la rambla del Pilar desde la última presa hasta su incorporación en el entramado urbano. Los cálculos recogidos en dicho informe, se han basado en el método racional para cuencas inferiores a $50 \mathrm{~km}^{2}$ que, teniendo en cuenta estas obras mencionadas, y el cálculo realizado, los resultados que obtienen es un calado de 1,2 y $4 \mathrm{~m}^{3} / \mathrm{s}$, para los periodos de retorno de 10, 100 y 500 años, respectivamente. Valores que no corresponden con la realidad vivida en el episodio de septiembre de 2019, además de que el sistema de presas está completamente colmatado, por lo que el caudal y las consecuencias vividas fueron mayores que los valores estimados en el SNCZI (Cuenca 19.8 Cañada de la Plana). Atendiendo a los periodos de retorno, se podría señalara que el caudal introducido en el mapa de peligrosidad (150), corresponderían a un periodo de retorno de 500 años (Figura 8).

Por su parte, la cartografía del PATRICOVA resulta mucho más genérica pero más completa, en lo que respecta a la peligrosidad de inundación para Callosa del Segura. Como se puede apreciar en la Figura 9 , el núcleo urbano de dicho municipio se vería afectado por una peligrosidad 4, asociada a un periodo de retorno de 100 años, y un caudal inferior a $0.8 \mathrm{~m}$, para la rambla del Pilar. Seguidamente, se encuentra la peligrosidad 2, asociada a un periodo de retorno comprendido entre 25 y 100 años, y un caudal superior a $0.8 \mathrm{~m}$ para la zona de huerta de Callosa, además, de parte del núcleo urbano en la zona más baja del cono aluvial o ya en la propia huerta. No obstante, conviene matizar que la capa de peligrosidad 2 corresponde 
Olcina Cantos, J., Oliva Cañizares, A., Sánchez Almodóvar, E., Martí Talavera, J., Biener Camacho, S. (2021). Cartografías para la acreditación del riesgo de inundaciones: SNCZI y PATRICOVA en la Comunidad Valenciana (España). GeoFocus (Artículos), Revista Internacional de Ciencia y Tecnología de la Información Geográfica, 27, 19-53. http://dx.doi.org/10.21138/GF.691

a una inundación masiva producida por el desbordamiento del río Segura, más que por la rambla del Pilar, objeto de estudio.

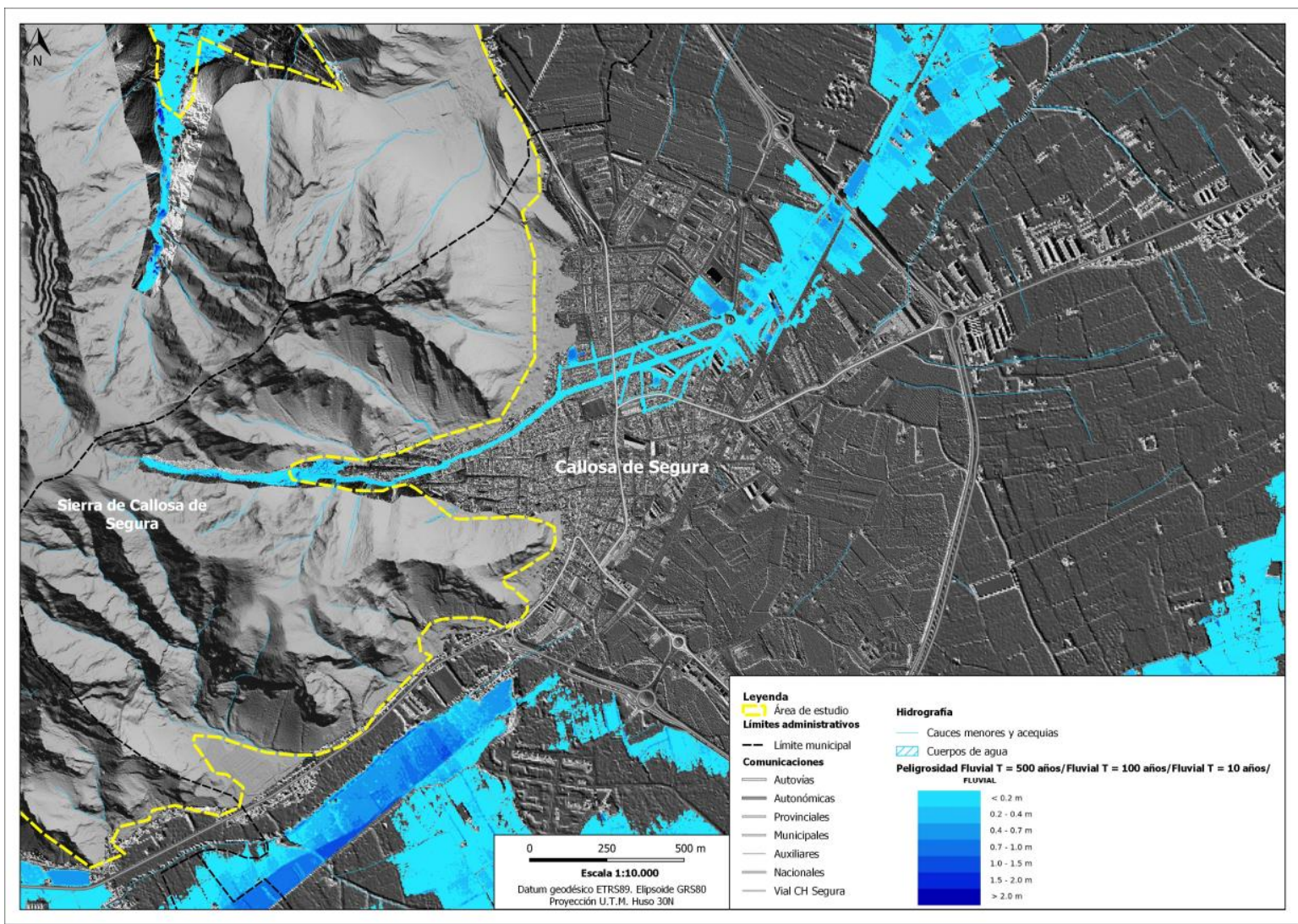

Figura 8. Mapa de peligrosidad según el SNCZI para Callosa del Segura.

Fte.: SNCZI, IGN e ICV. Elaboración propia.

Por último, no hay que olvidar la capa de peligrosidad geomorfológica del PATRICOVA. Ésta señala que las zonas anegadas corresponden con el sector norte del casco urbano, su zona de huerta norte, motivada por la inundación de un abanico torrencial, teniendo en cuenta la rambla del Pilar y la rambla de Tatus.

La suma de estas tres peligrosidades se asemeja a los resultados obtenidos en la elaboración del mapa de peligrosidad, aunque de manera genérica sin llegar a una mayor precisión o escala de detalle de la inundación, en función del discurrir de las aguas.

Atendiendo a los resultados de caudales del PATRICOVA, el caudal empleado en el mapa de peligrosidad (150) correspondería con un nivel de peligrosidad 2 y 4, asociado a un periodo de retorno entre 25 y 100 años ya que, según el punto donde se mida el calado de las aguas, puede ser superior (huerta) o inferior (rambla del Pilar) a $0.80 \mathrm{~m}$. 
Olcina Cantos, J., Oliva Cañizares, A., Sánchez Almodóvar, E., Martí Talavera, J., Biener Camacho, S. (2021). Cartografías para la acreditación del riesgo de inundaciones: SNCZI y PATRICOVA en la Comunidad Valenciana (España). GeoFocus (Artículos), Revista Internacional de Ciencia y Tecnología de la Información Geográfica, 27, 19-53. http://dx.doi.org/10.21138/GF.691

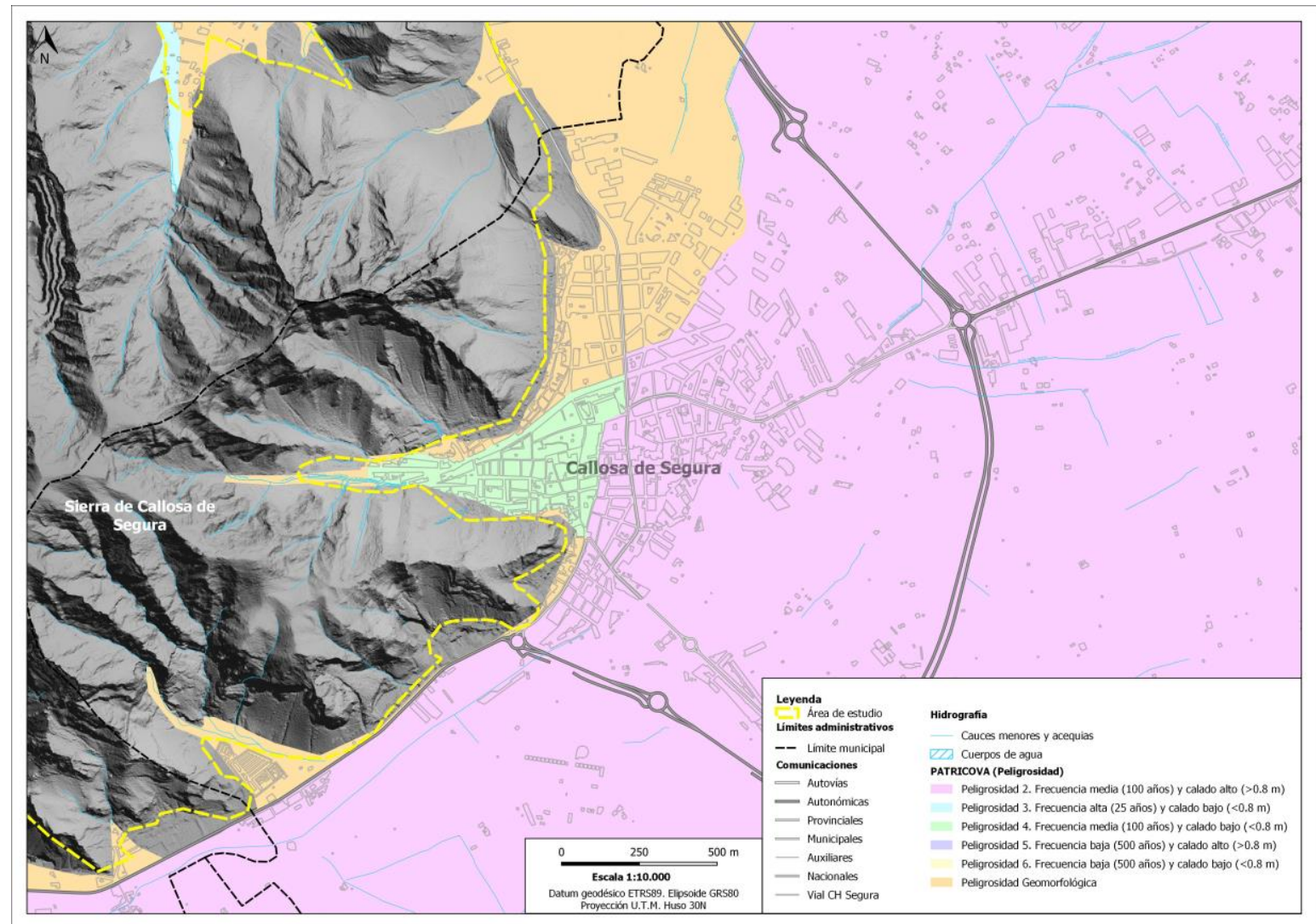

Figura 9. Mapa de peligrosidad según el SNCZI para Callosa del Segura.

Fuente: SNCZI, IGN e ICV. Elaboración propia.

Otro aspecto importante a considerar para la revisión de las cartografías oficiales, tiene que ver con el famoso debate sobre los periodos de retorno, que han abordado diferentes autores desde dos posiciones, a favor (McCuen, 1998; Flaming et al., 2002; Gupta, 2002) y en contra (Yue \& Rasmussen, 2002; Ruiz \& Núñez, 2011; Serinaldi, 2014; Doménech, Ollero \& Sánchez, 2015; Olcina \& Oliva, 2020). En la coyuntura actual de los efectos que se evidencia sobre el cambio climático en las regiones mediterráneas, sobre todo en el sureste peninsular, los cálculos probabilísticos basados en los periodos de retorno están desfasados y ya no son idóneos, ya que los cálculos matemáticos basados en fórmulas y ecuaciones ingenieriles no reflejaban ni reflejan la realidad atmosférica ni territorial en la que se halla la Comunidad Valenciana. De hecho, para la Vega Baja, se señala en el documento de MPRI del SNCZI, que el río Segura presentaría un caudal de 238, 940 y $1950 \mathrm{~m}^{3} / \mathrm{s}$, para los periodos de retorno de 10,100 y 500 años. Sin embargo, tras la inundación de septiembre de 2019, la cartografía de zonas inundables del SNCZI actualizó las zonas afectadas en 2020, y asociaron dicha inundación para un periodo de retorno de 500 años, hecho que no se cumple ya que el caudal del río Segura era entorno a los $450 \mathrm{~m}^{3} / \mathrm{s}$, y como éste, numerosos ejemplos más. 


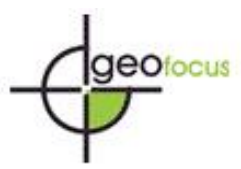

Olcina Cantos, J., Oliva Cañizares, A., Sánchez Almodóvar, E., Martí Talavera, J., Biener Camacho, S. (2021). Cartografías para la acreditación del riesgo de inundaciones: SNCZI y PATRICOVA en la Comunidad Valenciana (España). GeoFocus (Artículos), Revista Internacional de Ciencia y Tecnología de la Información Geográfica, 27, 19-53. http://dx.doi.org/10.21138/GF.691

Ello evidencia la necesidad de cambiar el paradigma empleado de cálculos matemáticosprobabilísticos, por cálculos posibilísticos, que se asemejan más a la definición del término riesgo empleado por Olcina en el año 2004. Ello supondría conocer las cantidades máximas y mínimas de precipitación, en $24 \mathrm{~h}$, que se dan en el territorio y actuar conforme a ello. En el caso que atañe a la investigación, cabría conocer los valores máximos y mínimos de precipitación registrados en toda la Comunidad Valenciana, en las provincias y en las zonas de estudio. Sirva de ejemplo la diferencia de registros pluviométricos existentes en la provincia de Alicante, donde se conoce que en Oliva se registraron el máximo de precipitación acumulada en $24 \mathrm{~h}$ de toda España con $817 \mathrm{l} / \mathrm{m}^{2}$, en noviembre de 1987. Por su parte, en lo que corresponde a la zona de estudio en la Vega Baja, en el episodio de noviembre de 1987, se registraron más de $3001 / \mathrm{m}^{2}$, lo que supone la precipitación anual de la comarca, reducido en $24-48 \mathrm{~h}$.

Sin embargo, este récord de precipitación de la Vega Baja es superado por el episodio de septiembre de 2019, donde se registraron en algunos puntos una precipitación de más de $5001 / \mathrm{m}^{2}$, evidencia más que contrastada con datos científicos sobre los efectos del cambio climático la cuenca occidental del Mediterráneo, en el cual uno de ellos corresponde al incremento de episodios de lluvias torrenciales, concentrada en breves periodos de tiempo, lo que se traduce en registros pluviométricos de gran volumen e intensidad, generadores de episodios de inundación (CEDEX, 2012, Olcina, 2017 y 2019; Nelles \& Serrer, 2020). A ello, se le unen otras características que corroboran los efectos del cambio climático como lo son el calentamiento del Mar Mediterráneo o el incremento de la temperatura superficial del mar en épocas estivales y se prolongan los meses de otoño, donde el mar presenta temperaturas elevadas $\left(27-29^{\circ} \mathrm{C}\right)$ favorecedoras de la formación de precipitaciones intensas y torrenciales (Pastor, Valiente \& Palau, 2017; Pastor, 2020); la ralentización y meandrización del Jet Stream y los sucesivos descuelgues de gotas frías (Muñoz, Schultz \& Vaughan, 2020), entre otras, como el incremento de temperaturas medias, máximas y mínimas anuales (Olcina, 2019). Aspectos que, en la actualidad, ya se evidencian como efectos del cambio climático en la cuenca occidental del Mediterráneo.

Por consiguiente, se trata de tener en cuenta la posibilidad que el valor mínimo es 0 y el máximo es $817 \mathrm{l} / \mathrm{m}^{2}$ que, si se juntan los factores adecuados, pueden darse en cualquier parte de la provincia de Alicante. Si se reduce la escala de trabajo, a la Vega Baja del Segura, dichos valores oscilarían entre 0 y $520 \mathrm{l} / \mathrm{m}^{2}$. Asimismo, en el transcurso de treinta y dos años, desde la última riada en dicha comarca (1987), parece que los efectos del cambio climático han incrementado en $2001 / \mathrm{m}^{2}$, las precipitaciones que se pueden registrar en la provincia de Alicante. Por tanto, atendiendo a los valores máximos y mínimos registrados, los cálculos y medidas que se tomen deben considerar estas cuestiones e incluso, dar un margen mayor entre la precipitación máxima registrada y $150-2001 / \mathrm{m}^{2}$ más añadidos, para adaptarse a las inundaciones y al cambio climático. El problema reside que, ello supone una mayor inversión económica y la relación coste-beneficio sale costosísima, de manera que siempre se elige la opción más barata que solventan algunos problemas, empero, los más severos no por no realizar la inversión adecuada en el momento oportuno. De hecho, esto es una cuestión que preocupa bastante en el ámbito europeo y que se recoge en el informe PESETA se preocupan por esta cuestión porque si no se toman medidas de adaptación a las inundaciones y al cambio climático, en la actualidad, dentro de unos años serán más costosas.

Todas estas consideraciones deben ser incluidas en la próxima revisión del PATRICOVA que debe ser aprobada en el año 2023 y, probablemente, se publiquen los resultados finales en 2025. Lo que implica que durante los próximos diez años (2023-2033), la cartografía que se determine implicará la permisividad 
Olcina Cantos, J., Oliva Cañizares, A., Sánchez Almodóvar, E., Martí Talavera, J., Biener Camacho, S. (2021). Cartografías para la acreditación del riesgo de inundaciones: SNCZI y PATRICOVA en la Comunidad Valenciana (España). GeoFocus (Artículos), Revista Internacional de Ciencia y Tecnología de la Información Geográfica, 27, 19-53. http://dx.doi.org/10.21138/GF.691

o no de determinados usos, que se traducen en una mayor o menor exposición y, por ende, en un incremento o no del riesgo, o de la resiliencia del territorio.

Por consiguiente, durante una década no se volverá a realizar la revisión de esta cartografía, aunque es aconsejable realizar alguna actualización cada tres años o cada lustro, hasta los diez años donde se realizaría una revisión y actualización mucho más completa- por lo que la vida de las personas estarán supeditadas al trazado de los criterios contemplados por los técnicos, los resultados de mapas de peligrosidad y de riesgo de inundación, así como de la normativa reguladora de usos en zonas inundables (normas urbanísticas). Bajo esta tesitura, impera la necesidad urgente de incluir estos aspectos para reducir los daños económicos y prevaleciendo, sobre todo, por encima de los daños económicos, las vidas humanas. La justificación de ello se basa en que las pérdidas económicas, en ocasiones, suelen ser recuperables, pero las vidas humanas no. Es por ello que llega el momento de dar un paso más allá y tener en cuenta estas cuestiones.

A estos aspectos señalados se le une la problemática que presenta el cambio climático por acción antrópica, donde las fechas señaladas implican el comienzo de los escenarios sobre los efectos del calentamiento global a corto plazo (2030), contemplados en los escenarios futuros por el RCP 4.5 y el RCP 8.5, en la cual existen numeroso estudios que señalan que el ámbito mediterráneo es uno de los espacios más vulnerables a los efectos del cambio climático y cuyas consecuencias serán más evidentes todavía que en la actualidad (IPCC, 2013-2014, 2018 y 2019; JRC, 2018 y JRC, 2020; MedECC, 2020; MITECO, 2020a y 2020b; AEMET, 2020; Olcina, 2019). Es por ello por lo que se considera necesario y obligatorio incorporar, con carácter urgente, estas cuestiones en los mapas de riesgo naturales, para estar preparados ante las incertidumbres que puedan suceder. Por consiguiente, las cartografías oficiales van a jugar un rol fundamental para que, conociendo y ordenando el territorio, conviertan el territorio español en un espacio resiliente y adaptado al cambio climático y a las inundaciones.

Estos hechos están apoyados y contrastados en los informes del IPCC (2013-2014, 2018 y 2019) y del MedECC (2020). Asimismo, los informes europeos sobre inundaciones y cambio climático señalan que no tomar las medidas adecuadas para la adaptación a las inundaciones y al cambio climático, supondrá unas mayores pérdidas económicas y sociales que si se actúa (JRC, 2018; JRC, 2020). En este sentido, y como se ha señalado a lo largo de la investigación, la cartografía del riesgo debe ser la principal herramienta para los procesos de ordenación del territorio y, en una coyuntura como la actual, que la visión europea no apuesta por las medidas "grises", pero sí en las medidas preventivas y en las infraestructuras verdes, mayor importancia adquiere la cartografía como herramienta esencial en materia de ordenación del territorio, cuya finalidad es imprescindible: la salvaguarda de las vidas humanas.

\section{Conclusiones}

La cartografía de riesgo es una herramienta fundamental y de acreditación de riesgos naturales (inundaciones) para la elaboración de los planes de ordenación territorial y de protección civil y gestión de emergencias. En España, este tema adquiere una gran importancia con la aprobación de la Ley del Suelo de 2008, donde se obliga a la elaboración de un mapa de riesgos naturales (en plural) para cualquier tipo de actuación urbanística. De esta manera el mapa permite acreditar un riesgo de manera jurídica, permitiendo o prohibiendo una serie de determinados usos en función de su regulación. 
Olcina Cantos, J., Oliva Cañizares, A., Sánchez Almodóvar, E., Martí Talavera, J., Biener Camacho, S. (2021). Cartografías para la acreditación del riesgo de inundaciones: SNCZI y PATRICOVA en la Comunidad Valenciana (España). GeoFocus (Artículos), Revista Internacional de Ciencia y Tecnología de la Información Geográfica, 27, 19-53. http://dx.doi.org/10.21138/GF.691

En lo que respecta a España y a la Comunidad Valenciana, existen dos cartografías de riesgo de inundación oficial, el SNCZI y el PATRICOVA, respectivamente. La metodología empleada en su desarrollo ha sido diferente, no obstante, en la mayor parte de la Comunidad Valenciana, coinciden respecto a las zonas inundables. Conviene destacar que, a diferencia del SNCZI, el PATRICOVA posee la denominada capa de peligrosidad geomorfológica que detalla y complementa los niveles de peligrosidad hidrológica. Sin embargo, a la hora de elaborar los mapas de riesgo de inundación, tanto el SNCZI como el PATRICOVA, emplean las capas de peligrosidad de inundación, obviando la geomorfológica.

Con el análisis empleado en el presente trabajo, se llega a la conclusión de que, la capa de peligrosidad geomorfológica, también debe ser incorporada en la elaboración de los mapas de riesgo de inundación, cuyo resultado mostraría las zonas inundables y zonas de afección por estas morfologías que, como aconteció en el episodio de septiembre de 2019 en la Vega Baja del Segura, tienen un alto impacto sobre la población y en la agricultura del área de estudio.

Además, si se tiene en cuenta los escenarios futuros del cambio climático, con el incremento de los fenómenos meteorológicos de rango extraordinario, se hace más que necesario la incorporación de los aspectos geomorfológicos para los próximos mapas de riesgo, ya que lo elementos que lo conforman afectan directamente a la población, lo que supone un riesgo elevado para las vidas humanas.

Por estos motivos, y ante la próxima revisión de la cartografía del PATRICOVA, se propone la incorporación de los espacios que presentan peligrosidad geomorfológica para la elaboración de los mapas de riesgo. Asimismo, se plantea una clasificación metodológica diferente, pero complementaria a la existente, basada en los niveles de calados y la velocidad de las aguas de inundación, en función del riesgo que supone para las vidas humanas. El objetivo de la metodología propuesta es conseguir mostrar un riesgo "real" para las personas, que permita la salvaguarda de las vidas humanas; así como tratarse de un aspecto complementario a las cartografías actuales basadas en los daños económicos.

Por consiguiente, y atendiendo a la jerarquía escalar, los primeros mapas a consultar y a tener en cuenta, tanto para la peligrosidad como para el riesgo de inundación, es el SNCZI que, seguidamente, debe complementarse - para la Comunidad Valenciana- con la cartografía del PATRICOVA. En el caso de que, en un espacio de dicha autonomía, no existiera cartografía del SNCZI, se debe consultar el PATRICOVA -incluyendo la peligrosidad geomorfológica, para la peligrosidad y riesgo de inundación-, que se complementará con estudios inundables complementarios y más detallados. Sin embargo, si en un espacio no existe cartografía ni del SNCZI ni del PATRICOVA, se debe realizar un estudio de inundabilidad detallado y adecuado, a la normativa establecida por el PATRICOVA.

Por último, conviene destacar que, desde la aprobación de la Directiva 60/2007/CE sobre la evaluación y gestión del riesgo de inundaciones, la Unión Europea no ha realizado ningún informe de estado de cuestión, casi quince años después, en el cual, el continente europeo ha experimentado numerosas inundaciones, sobre todo los países mediterráneos. Esta cuestión, se plantea como una futura línea de trabajo o de investigación que resulta clave para la comprensión y los efectos que se están dejando notar en la Unión Europea y en los países mediterráneos, sobre el cambio climático. 


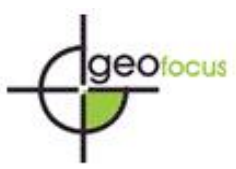

Olcina Cantos, J., Oliva Cañizares, A., Sánchez Almodóvar, E., Martí Talavera, J., Biener Camacho, S. (2021). Cartografías para la acreditación del riesgo de inundaciones: SNCZI y PATRICOVA en la Comunidad Valenciana (España). GeoFocus (Artículos), Revista Internacional de Ciencia y Tecnología de la Información Geográfica, 27, 19-53. http://dx.doi.org/10.21138/GF.691

\section{Agradecimientos}

Este artículo se inserta en una Beca predoctoral de Formación de Personal Universitario del Programa Propio del Vicerrectorado de Investigación para el Fomento de la I+D+I en la Universidad de Alicante (UAFPU2019-54) cuya beneficiaria es la coautora Esther Sánchez Almodóvar

\section{Referencias bibliográficas}

Agencia Estatal de Meteorología (AEMET) (2020): Proyecciones climáticas para el siglo XXI en España. [Consulta 15-10-2020]. Disponible en http://www.aemet.es/es/serviciosclimaticos/cambio climat

Camarasa-Belmonte, A. Caballero López, M. (2018): "Lluvias in situ en la Comunidad Valenciana. Relación entre indicadores pluviométricos, llamadas al centro de coordinación de emergencias (112) y relación de daños, durante el episodio de 26-30 de noviembre de 2016". En: Montávez J. P. Gómez Navarro, J. J. López Romero J. M. Palacios Peña, L. Turco, M. Jerez S. ... Meseguer Ruiz O. (Eds.). El clima: aire, agua, tierra y fuego. XI Congreso de la Asociación Española de Climatología, pp. 233-244. Disponible en http://hdl.handle.net/20.500.11765/9904

Centro de Estudios y Experimentación de Obras Públicas (CEDEX) (2012): Estudio de los impactos del cambio climático en los recursos hídricos y las masas de agua. [Consulta 4-10-2020]. Disponible en https://doi.org/10.1017/CBO9781107415324.004

Conesa García, C. (1995). Magnitud y frecuencia de sucesos hidromofológicos del Bajo Segura anteriores a su encauzamiento. Papeles de Geografía, O(22), 67-86.

Confederación Hidrográfica del Segura (CHS) (2019). Plan de gestión del Riesgo de Inundación de la Demarcación Hidrográfica del Segura. Ministerio para la Transición Ecológica y el Reto Demográfico (MITECO) [Consulta 12-04-2020]. Disponible https://www.chsegura.es/es/cuenca/caracterizacion/zonas-inundables-y-gestion-del-riesgo/plan-degestion-del-riesgo-de-inundacion-pgri/

CHS. (2020). Cronología de riadas en la cuenca del Segura. https://www.chsegura.es/es/confederacion/unpocodehistoria/cronologia-de-riadas-en-la-cuenca-del-

Segura/

Consellería d’Habitatge (2015): Plan de Acción Territorial sobre prevención del Riesgo de Inundación en la Comunitat Valenciana (PATRICOVA). Memoria. Valencia, Generalitat Valenciana. [Consulta 9-122020] Disponible en http://politicaterritorial.gva.es/documents/20551069/162377494/01+Memoria/9938fc0e-3ba8-4829-85245d3afa3542eb

Cutter, S. L., Boruff, B. J., \& Shirley, W. L. (2003). Social Vulnerability to Environmental Hazards. Social Science Quarterly, 84(2), 242-261. https://doi.org/10.1111/1540-6237.8402002

Cutter, S. L., Mitchell, J. T., \& Scott, M. S. (2000). Revealing the vulnerability of people and places: A case study of georgetown county, South Carolina. Annals of the Association of American Geographers, 90(4), 713-737. https://doi.org/10.1111/0004-5608.00219 


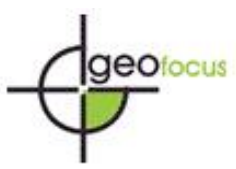

Olcina Cantos, J., Oliva Cañizares, A., Sánchez Almodóvar, E., Martí Talavera, J., Biener Camacho, S. (2021). Cartografías para la acreditación del riesgo de inundaciones: SNCZI y PATRICOVA en la Comunidad Valenciana (España). GeoFocus (Artículos), Revista Internacional de Ciencia y Tecnología de la Información Geográfica, 27, 19-53. http://dx.doi.org/10.21138/GF.691

Davoine, P-A. Arnaud, A. Gensel, J. Martin (2020): A tool for historical cartography about Natural Hazards [Consulta: 14-10-2020]. Disponible en https://citeseerx.ist.psu.edu/viewdoc/download?doi=10.1.1.600.733\&rep=rep1\&type=pdf

Doménech Zueco, S. Ollero Ojeda, A. Sánchez Fabre, M. (2015): "Núcleos de población en riesgo de inundación fluvial en Aragón: Diagnóstico y evaluación para la ordenación del territorio", Geographicalia, 54, pp. 17-44. https://doi.org/10.26754/ojs geoph/geoph.2008541095

EXCIMAP (2007). Handbook on good practices for flood mapping in Europe. European Exchange Circle on Flood Mapping.

Flaming, G. Frost, L. Huntington, S. Knight, D. Law, F. \& Rickard, C. (2002): Flood risk management: learning to live with rivers. London, Institution of Civil Engineers.

Gallegos Reina, A. Perles Roselló, M. J. (2020): "Methodology for the integrated analysis of hazards associated to flooding: proposal adapted to the spatian planning in Mediterranean regions", Boletín de la Asociación de Geógrafos Españoles (BAGE), 86, 46 p. https://doi.org/10.21138/bage.2950

Gupta, S.K. (2011): Modern hidrology and sustainable water development. Wiley, Chichester.

Intergovernmental Panel on Climate Change (IPCC) (2014): Climate Change 2013: The Physical Science Basis. Contribution of Working Group I to the Fifth Assesment Report of the Intergovernmental Panel on Climate Change.

IPCC (2018): Special Report on Global Warming of $1.5^{\circ} \mathrm{C}$. Contribution of Working Group I to the Fifth Assesment Report of the Intergovernmental Panel on Climate Change (AR5). [Consulta: 8-1-2021] Disponible en https://www.ipcc.ch/sr15/

IPCC (2019): Special Report on Climate Change, Desertification, Land Degradation, Sustainable Land Management, Food Security, and Greenhouse gas fluxes in Terrestrial Ecosystems. Contribution of Working Group I to the Fifth Assesment Report of the Intergovernmental Panel on Climate Change (AR5). [Consulta 8-1-2021]. Disponible en https://www. ipcc.ch/report/srccl/

Joint Research Centre (JRC) (2018): PESETA III. Task-7 River floods. European Commission, Luxembourg: Publications Office of the European Union. https://doi.org/10.2760/849948

JRC (2020): PESETA IV. Analysis of Events Related to Flooding at NPPs. European Commission, Luxembourg: Publications Office of the European Union. https://doi.org/10.2760/289502

Kellens, W. Vanneuvile, W. Ooms, K. de Maeyer, P. (2009): "Communicating flood risk to the public by cartography". $\quad 11$ p. [Consulta 10-1-2021]. Disponible en https://www.researchgate.net/publication/228676546 Communicating flood risk to the public by cart ography

Lane, S. N., Landström, C., \& Whatmore, S. J. (2011). Imagining flood futures: Risk assessment and management in practice. Philosophical Transactions of the Royal Society A: Mathematical, Physical and Engineering Sciences, 369(1942), 1784-1806. https://doi.org/10.1098/rsta.2010.0346

McCuen, R.H. (1998): Hydrologic analysis and designs. Prentice Hall, NJ, 2nd ed, Springer, New York. 


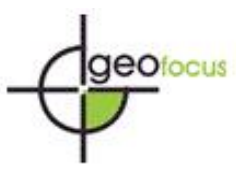

Olcina Cantos, J., Oliva Cañizares, A., Sánchez Almodóvar, E., Martí Talavera, J., Biener Camacho, S. (2021). Cartografías para la acreditación del riesgo de inundaciones: SNCZI y PATRICOVA en la Comunidad Valenciana (España). GeoFocus (Artículos), Revista Internacional de Ciencia y Tecnología de la Información Geográfica, 27, 19-53. http://dx.doi.org/10.21138/GF.691

Mediterranean Experts on Climate and environmental Change (MedECC) (2020): "Climate and Environmental Change in the Mediterranean Basin - Current Situation and Risks for the Future". First Mediterranean Assessment Report (MAR1) [Cramer, W, Guiot, J., Marini, K. (eds.)] Union for the Mediterranean, Plan Bleu, UNEP/MAP, Marseille, France, 600 p.

Ministerio de Agricultura y Pesca, Alimentación y Medio Ambiente (2017): Guía técnica de apoyo a la aplicación del reglamento del dominio público hidráulico en las limitaciones a los usos del suelo en las zonas inundables de origen fluvial, Madrid, $74 \mathrm{p}$.

Ministerio de Fomento. (2015): Real Decreto Legislativo 7/2015, de 30 de octubre, por el que se aprueba el texto refundido de la Ley de Suelo y Rehabilitación Urbana. Boletín Oficial Del Estado, 261, 31 de octubre, 55. https://www.boe.es/eli/es/rdlg/2015/10/30/7/con

Ministerio para la Transición Ecológica (2019): Recomendaciones para la construcción y rehabilitación de edificaciones en zonas inundables, Madrid, $74 \mathrm{p}$.

Ministerio para la Transición Ecológica (2019): Propuesta de mínimos para la realización de los mapas de riesgo de inundación. Directiva de Inundaciones, $2^{\circ}$ ciclo, Madrid, 97 p.

Ministerio para la Transición Ecológica y el Reto Demográfico (2020a): Adaptación al Cambio Climático en España. [Consulta 10-1-2021]. Disponible en https://adaptecca.es/

Ministerio para la Transición Ecológica y el Reto Demográfico (202b): Plan Nacional de Adaptación al Cambio Climático (2021-2030) (PNACC). Madrid, Ministerio para la Transición Ecológica y el Reto Demográfico, $246 \mathrm{p}$.

Muñoz, C. Schultz, D. Vaughan, G. (2020). A Midlatitude Climatology and Interannual Variability of 200- and 500hPa Cut-Off Lows, Journal Of Climate, 33(6), pp. 2201-2222. Disponible en https://journals.aemetsoc.org/view,journals/clim/33/6/jcli-d-19-0497.1.xml

Nelles, D. Serrer, C (2020): El pequeño manual del cambio climático. Grijalbo Ilustrados, $1^{\text {a }}$ ed., 127 p.

Olcina Cantos, J. (2017): "Incremento de episodios de inundación por lluvias de intensidad horaria en el sector central del litoral Mediterráneo español: análisis de tendencia en Alicante", Sémata, Ciencias Sociales y Humanidades, 29, pp. 143-163.

Olcina Cantos, J. (2018) “¿Es la ordenación del territorio una medida eficaz contra el riesgo de inundaciones en España?", en Riesgos naturales y Derecho: una perspectiva interdisciplinar (Arana García, E., dir.), Madrid, Editorial Dykinson, p. 63-82.

Olcina Cantos, J. (2019): "Evidencias e incertidumbres del cambio climático y de los riesgos asociados en el litoral mediterráneo español”, Boletín de la Real Sociedad Geográfica, CLIV, 9-34 pp.

Olcina Cantos, J. Díez-Herrero, A. (2017): "Cartografía de inundaciones en España", Estudios Geográficos, 78 (282), p. 283-315. https://doi.org/10.3989/estgeogr.201710

Olcina Cantos, J. Oliva Cañizares, A. (2020): "Medidas estructurales versus cartografía de inundación en la valoración del riesgo en áreas urbanas: El caso del barranco de las Ovejas (Alicante, España)", Cuadernos Geográficos, 59 (2), 199-220 pp.

Oliva Cañizares, A. Sainz-Pardo Trujillo, A. Sánchez Almodóvar, E. (2020): "La cartografía de 
Olcina Cantos, J., Oliva Cañizares, A., Sánchez Almodóvar, E., Martí Talavera, J., Biener Camacho, S. (2021). Cartografías para la acreditación del riesgo de inundaciones: SNCZI y PATRICOVA en la Comunidad Valenciana (España). GeoFocus (Artículos), Revista Internacional de Ciencia y Tecnología de la Información Geográfica, 27, 19-53. http://dx.doi.org/10.21138/GF.691

vulnerabilidad como base de los Planes de Emergencia: análisis-diagnóstico del término municipal de Daya Vieja (Alicante)". En López Ortiz, M, I. y Melgarejo Moreno, J (eds.). Riesgo de Inundación en España: análisis y soluciones para la generación de territorios resilientes, pp. 333-345. Alicante, Universidad de Alicante.

Pastor, F., Valiente, J. A., Palau, J. L. (2017): "Sea Surface Temperatura in the Mediterranean: Trends and Spatial Patterns (1982-2016)" (Centros de Estudios Ambientales del Mediterráneo, CEAM), Pure and Applied Geophysics, 175, 4017-4029 pp. https://doi.org/10.1007/s00024-017-1739-Z

Pastor, F. (2020): Mediterranean SST report (Autumn 2020). Paterna, (Centros de Estudios Ambientales del Mediterráneo, CEAM) https://doi.org/10.13140/RG.2.2.36799.94883

Pérez Morales, A., Navarro Hervás, F., \& Álvarez Rogel, Y. (2016). Propuesta metodológica para la evaluación de la vulnerabilidad social en poblaciones afectadas por el peligro de inundación: el caso de Águilas (Murcia, sureste ibérico). Documents d'Anàlisi Geogràfica, 62(1), 133. https://doi.org/10.5565/rev/dag.242

Perles Roselló, Mª J. Olcina Cantos, J. Mérida Rodríguez, M. (2018): "Balance de las políticas de gestión del riesgo de inundaciones en España: de las acciones estructurales a la ordenación territorial”, Ciudad y Territorio. Estudios Territoriales, p. 417-438.

Romero Díaz, A., \& Maurandi Guirado, A. (2000). Las inundaciones en la cuenca del Segura en las dos últimas décadas del siglo XX: actuaciones de prevención. Serie geográfica, 9, 93-120. http://hdl.handle.net/10017/1096

Ruíz García, J. A. Núñez Mora, J. A. (2011): "Sobre los periodos de retorno de las precipitaciones extraordinarias en la Comunidad Valenciana". Agencia Estatal de Meteorología (AEMET): Calendario Meteorológico, 2012, pp. 265-273. Disponible en http://hdl.handle.net/20.500.11765/2465

Sanchez-Rubio, C. J., Ponce Herrero, G., \& Canales Martínez, G. (1989). Inundaciones en el Bajo Segura. Cronología de una lucha intermitente frente a una amenaza constante (1946-1987). En A. Gil Olcina \& A. Morales Gil (Eds.), Avenidas fluviales e inundaciones en la Cuenca del Mediterráneo (pp. 309-329). Instituto Universitario de Geografía de la Universidad de Alicante y Caja de Ahorros del Mediterráneo.

Serinaldi, F. (2015): “Dismissing return periods!”, Springer, Stach Environ Res Risk Acces, 29, pp. 11791189. https://doi.org/10/1007/s00477-014-0916-1

Soriano García, J. Sanchis-Ibor, C. Camarasa-Belmonte, A. (2020): "Integración de cartografías de inundabilidad en la Comunidad Valenciana (PATRICOVA y SNCZI)". En Crisis y espacios de oportunidad. Retos para la Geografía. XXVI Congreso de la Asociación Española de Geografía. Valencia, pp. 211-223.

Sortino Barrionuevo, J. F., \& Perles Roselló, M. J. (2017). Metodología para una cartografía de vulnerabilidad del territorio frente al riesgo de inundación relacionado con los sistemas de socorro y asistencia. Aplicación al área inundable del bajo Guadalhorce (2016). Papeles de Geografía, Número monográfico del XV Coloquio Ibérico de Geografía, 157-178. https://doi.org/https://doi.org/10.6018/geografia/2017/307051

Yue, S. Rusmussen, P. (2002): "Bivariate frequency analysis: discussion of some useful concepts in hydrological application”, Hydrol Process, 16, pp. 2881-2898. 


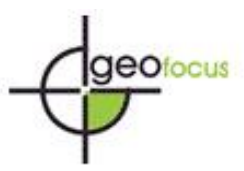

Olcina Cantos, J., Oliva Cañizares, A., Sánchez Almodóvar, E., Martí Talavera, J., Biener Camacho, S. (2021). Cartografías para la acreditación del riesgo de inundaciones: SNCZI y PATRICOVA en la Comunidad Valenciana (España). GeoFocus (Artículos), Revista Internacional de Ciencia y Tecnología de la Información Geográfica, 27, 19-53. http://dx.doi.org/10.21138/GF.691

Whatmore, S. J. (2009). Mapping knowledge controversies: science, democracy and the redistribution of expertise. Progress in Human Geography, 33(5), 587-598. https://doi.org/10.1177/0309132509339841

Whatmore, S. J., \& Landström, C. (2011). Flood apprentices: an exercise in making things public. Economy and Society, 40(4), 582-610. https://doi.org/10.1080/03085147.2011.602540 
